\title{
Interaction of antibacterial compounds with RND efflux pumps in Pseudomonas aeruginosa
}

\author{
Jürg Dreier ${ }^{*}$ and Paolo Ruggerone ${ }^{2}$ \\ ${ }^{1}$ Basilea Pharmaceutica International Ltd., Basel, Switzerland, ${ }^{2}$ Dipartimento di Fisica, Università di Cagliari - Cittadella \\ Universitaria, Monserrato, Italy
}

Pseudomonas aeruginosa infections are becoming increasingly difficult to treat due to intrinsic antibiotic resistance and the propensity of this pathogen to accumulate diverse resistance mechanisms. Hyperexpression of efflux pumps of the ResistanceNodulation-Cell Division (RND)-type multidrug efflux pumps (e.g., MexAB-OprM), chromosomally encoded by mexAB-oprM, mexCD-oprJ, mexEF-oprN, and mexXY

OPEN ACCESS

Edited by:

Klaas Martinus Pos, Goethe University Frankfurt, Germany

Reviewed by:

Aixin Yan,

The University of Hong Kong,

Hong Kong

Yuji Morita,

Aichi Gakuin University, Japan

Etinosa Igbinosa,

University of Benin, Nigeria

*Correspondence:

Jürg Dreier,

Basilea Pharmaceutica Internationa

Ltd., Grenzacherstrasse 487,

P.O. Box 4005, Basel, Switzerland

juerg.dreier@basilea.com

Specialty section:

This article was submitted to Antimicrobials, Resistance and Chemotherapy,

a section of the journal

Frontiers in Microbiology

Received: 13 February 2015

Accepted: 16 June 2015

Published: 08 July 2015

Citation:

Dreier $J$ and Ruggerone P (2015) Interaction of antibacterial compounds with RND efflux pumps

in Pseudomonas aeruginosa.

Front. Microbiol. 6:660.

doi: 10.3389/fmicb.2015.00660
(-oprA) is often detected in clinical isolates and contributes to worrying multi-drug resistance phenotypes. Not all antibiotics are affected to the same extent by the aforementioned RND efflux pumps. The impact of efflux on antibiotic activity varies not only between different classes of antibiotics but also between members of the same family of antibiotics. Subtle differences in physicochemical features of compoundpump and compound-solvent interactions largely determine how compounds are affected by efflux activity. The combination of different high-resolution techniques helps to gain insight into the functioning of these molecular machineries. This review discusses substrate recognition patterns based on experimental evidence and computer simulations with a focus on MexB, the pump subunit of the main RND transporter in $P$. aeruginosa.

Keywords: efflux, multi-drug resistance, bacteria, RND, substrate recognition, Pseudomonas aeruginsoa, antibiotic agents, efflux pump inhibitors

\section{Introduction}

Infectious diseases, including bacterial infections, are among the major causes of mortality worldwide (Mason et al., 2003). Infections by multi-drug resistant (MDR) pathogens are especially difficult to treat and are recognized as a major threat (CDC, 2013; Denys and Relich, 2014; Martinez and Baquero, 2014; ECDC, 2015).

The Gram-negative bacterium Pseudomonas aeruginosa is frequently involved in healthcareassociated infections like pneumonia, bloodstream infections, urinary tract infections, and surgical site infections (Hidron et al., 2008; Jones et al., 2009; Zhanel et al., 2010). About $8 \%$ of nosocomial infections reported to the Centers for Disease Control and Prevention in the US are ascribed to P. aeruginosa. $13 \%$ of the severe cases in the US are caused by MDR isolates and $14 \%$ of European isolates reported between 2005 and 2013 had combined resistance to several antibiotics (CDC, 2013; ECDC, 2015). MDR phenotypes have been described for clinical isolates from various places all over the world (Potron et al., 2015). MDR P. aeruginosa isolates are sometimes resistant to nearly all classes of antibiotics and have lost susceptibility toward fluoroquinolones, aminoglycosides, 
cephalosporins, and carbapenems (Livermore, 2009; Riou et al., 2010; Poole, 2011; Castanheira et al., 2014).

The outer membrane (OM) of Gram-negative bacteria has an asymmetric structure including an outer leaflet made of lipopolysaccharides (LPS), an inner phospholipid leaflet, and porin channels (Kamio and Nikaido, 1976; Nikaido, 2003). Small, hydrophilic compounds can pass the OM by diffusion through the porin channels whereas large and/or hydrophobic compounds have to go through the lipid bilayer (Hancock, 1997; Delcour, 2009). In the Escherichia coli OM, there are trimeric porins like $\mathrm{OmpF}$ and $\mathrm{OmpC}$ present that allow a relatively rapid diffusion of small, hydrophilic substances (Nikaido and Rosenberg, 1983; Cowan et al., 1992; Schulz, 1993). P. aeruginosa does not make such trimeric porins but expresses the monomeric porin OprF at a low number with a small opening that only allows slow permeation (Angus et al., 1982; Yoshimura and Nikaido, 1982; Sugawara et al., 2006, 2010). P. aeruginosa has also specific channels such as OprD for basic amino acids and peptides, which is the main entry passage of carbapenem antibiotics (Nikaido, 2003). The structure of the OM can be adapted by $P$. aeruginosa to decrease the net negative charge of the LPS in response to cationic peptides such as polymyxin $B$, which act on the negatively charged LPS (Olaitan et al., 2014). Thus, the $\mathrm{OM}$ of $P$. aeruginosa strongly reduces the permeability for most antibiotics and provides an effective and adaptable protection against antibacterial agents (Delcour, 2009; Page, 2012).

Many of the compounds that can pass through the $\mathrm{OM}$ are actively transported out of the cell again by efflux pumps. The low permeability of the OM combined with such efflux pumps results in an effective protection against a wide variety of substances including antibiotics (Kumar and Schweizer, 2005; Fernandez and Hancock, 2012). P. aeruginosa PAO1 has 12 efflux systems of the Resistance-Nodulation-Cell Division (RND) family (Poole, 2000, 2004, 2005, 2013; Webber and Piddock, 2003; Piddock, 2006; Zechini and Versace, 2009; Fernandez and Hancock, 2012; Nikaido and Pages, 2012; Blair et al., 2014, 2015b; Delmar et al., 2014; Sun et al., 2014), whereof a set of four RND pumps contributes most significantly to antibiotic resistance: MexAB-OprM, MexCD-OprJ, MexEF-OprN, and MexXY-OprM (Fernandez and Hancock, 2012). MexB transports $\beta$-lactams including $\beta$-lactamase inhibitors and carbapenems (not imipenem), aminoglycosides, fluoroquinolones, tetracyclines, tigecycline, macrolides, amphenicols, novobiocin, sulfonamides, trimethoprim, cerulenin, thiolactomycin, some amphiphilic molecules, disinfectants, dyes, solvents, detergents, and several homoserine lactones involved in quorum sensing [detailed lists of RND substrates are given in (Poole, 2005; Lister et al., 2009) and in Table 1 for dyes described in this review]. MexD recognizes fluoroquinolones, zwitterionic cephalosporins, macrolides, chloramphenicol, trimethoprim, and tetracyclines. MexF accepts fluoroquinolones, chloramphenicol, trimethoprim, and tetracycline as substrates. MexY transports aminoglycosides, fluoroquinolones, macrolides, tetracyclines, tigecycline, and zwitterionic cephalosporins (Morita et al., 2012).

The expression of RND pumps is regulated as a response to external stress factors such as reactive oxygen species (MexAB-OprM, MexXY-OprM), reactive nitrogen species
(MexEF-OprN), and other agents imposing stress to the bacterial cell like membrane damaging agents (MexCD-OprJ) or ribosome blocking substances (MexXY-OprM), (Grkovic et al., 2002; Lister et al., 2009; Morita et al., 2014; Poole, 2014). Thus, efflux pumps may be part of a versatile protection mechanism against cellular stress that works not only in response to naturally occurring signals but also against antibiotics.

Increased pump expression can be linked to decreased porin expression as for example in the $n f x C$ mutants of $P$. aeruginosa. In these mutants $\mathrm{OprD}$ expression is downregulated, which impairs carbapenem uptake and MexEF-OprN expression is upregulated, which affects fluoroquinolone export (Fukuda et al., 1990, 1995; Davin-Regli et al., 2008; Nishino et al., 2009; Castanheira et al., 2014).

One possibility to circumvent efflux is the development of antibiotics that are not pump substrates, or are only poorly affected by pump activity (e.g., Hayashi et al., 2014). Alternatively, one may look for molecules that inhibit pumps and can be used as adjuvants in combination with antibiotics (e.g., Olivares et al., 2013). For both strategies it is crucial to understand the molecular properties that define pump substrates. This review describes experimental procedures that are sensitive to RND efflux pump activity and allow conclusions on substrate recognition by RND efflux pumps. The described methods include specifically designed efflux assays but also assays which were developed for other purposes than efflux studies but revealed substrates of efflux pumps (e.g., probes for membrane integrity have been found to be efflux pump substrates). Substrate recognition by MexB from $P$. aeruginosa can often not (or not yet) be investigated with the methods described for AcrB in E. coli but can be modeled with in silico methods based on experimental data. Specific data about substrate recognition by MexB from $P$. aeruginosa are still limited. Therefore we discuss in detail results obtained also for AcrB when they describe similarities between the two RND transporters. It is paradigmatic that to date and to our knowledge there is only a single computational study addressing the molecular aspects of MexB-substrate interactions.

\section{Impact of Efflux on Antibiotic Activity}

A direct impact of efflux on antibiotic activity on P. aeruginosa was shown for a core set of RND pumps by efflux-pump deletion mutants and could be confirmed by mutants that overexpress selected RND systems (Li et al., 1995; Lomovskaya et al., 1999; Masuda et al., 2000a; Poole, 2000, 2004, 2005, 2013; Schweizer, 2003). Susceptibility of $P$. aeruginosa towards many antibiotics has been restored when the four systems that are most relevant for antibiotic resistance (MexAB-OprM, MexCD-OprJ, MexEFOprN, and MexXY-OprM) have been deleted (Morita et al., 2001; Kumar et al., 2006). These $P$. aeruginosa RND pumps have overlapping but not identical substrate ranges as mentioned in the Section Introduction. RND efflux pumps have individual substrate specificities that include amphiphilic molecules (e.g., MexB) but also hydrophobic solutes (e.g., MexB, MexD) and the hydrophilic polycationic aminoglycosides (MexY), described to enter cells by a self-promoted mechanism (Hancock, 1997). 
TABLE 1 | Properties of efflux-pump substrates and inhibitors.

\begin{tabular}{|c|c|c|c|c|c|c|c|}
\hline Compound & $\log P(o / w)^{a}$ & TPSA $\left(\AA^{2}\right)^{b}$ & $M W(D a)^{c}$ & $P C+{ }^{d}$ & PC-e & Net PC & Properties \\
\hline D13-9001 & 0.85 & 210.8 & 693.8 & 9.96 & -9.96 & 0 & Not transported \\
\hline Mefloquin & 4.27 & 49.7 & 379.3 & 6.13 & -5.13 & 1 & Quinolone \\
\hline \multicolumn{8}{|c|}{ Substrates acting as inhibitor ${ }^{9}$} \\
\hline Minocycline & -0.28 & 164.6 & 457.5 & 7.11 & -7.11 & 0 & Antibiotic \\
\hline Taurocholate & 2.33 & 141.0 & 514.7 & 4.79 & -5.79 & -1 & Bile acid \\
\hline Erythromycin & 2.76 & 195.1 & 734.9 & 9.73 & -8.73 & 1 & Antibiotic \\
\hline Glycocholate & 3.15 & 129.9 & 464.6 & 4.14 & -5.14 & -1 & Bile acid \\
\hline \multicolumn{8}{|l|}{ Substrates } \\
\hline Ceftobiprole & -1.29 & 213.7 & 533.6 & 9.53 & -10.5 & -1 & Antibiotic \\
\hline Aztreonam & -1.03 & 201.3 & 433.4 & 7.09 & -9.10 & -2 & Antibiotic \\
\hline FDG & -0.40 & 234.3 & 656.6 & 10.38 & -10.4 & 0 & Intracellular conversion into a fluorescent product \\
\hline Moxalactam & -0.38 & 212.0 & 518.5 & 7.60 & -9.60 & -2 & Antibiotic \\
\hline Tigecycline & -0.26 & 210.3 & 586.7 & 9.99 & -8.99 & 1 & Antibiotic \\
\hline Cefamandole & -0.18 & 153.4 & 461.5 & 6.47 & -7.47 & -1 & Antibiotic \\
\hline (+)-Cerulenin & 0.10 & 72.7 & 223.3 & 3.39 & -3.39 & 0 & Antibiotic \\
\hline Cefsulodine & 0.21 & 187.7 & 531.5 & 9.08 & -10.1 & -1 & Antibiotic \\
\hline Gemifloxacin & 0.36 & 125.8 & 389.4 & 6.80 & -6.81 & 0 & Antibiotic \\
\hline Doxorubicin & 0.51 & 207.7 & 544.5 & 8.89 & -7.89 & 1 & Anticancer drug, quenched intracellular fluorescence \\
\hline Cephalotin & 0.58 & 115.8 & 395.4 & 5.48 & -6.48 & -1 & Antibiotic \\
\hline Sulbenicillin & 0.59 & 140.8 & 412.4 & 5.97 & -7.97 & -2 & Antibiotic \\
\hline Dodecyl- $\alpha$-D-maltoside & 0.70 & 178.5 & 510.6 & 7.00 & -7.00 & 0 & Detergent \\
\hline Ceftazidime & 1.35 & 194.1 & 545.6 & 8.68 & -9.68 & -1 & Antibiotic \\
\hline Resazurin & 1.79 & 75.3 & 229.2 & 2.80 & -2.81 & 0 & Intracellular conversion into a fluorescent product \\
\hline Ala-NAP & 1.88 & 56.7 & 215.3 & 4.02 & -3.02 & 1 & Intracellular conversion into a fluorescent product \\
\hline Cefaloridine & 1.96 & 93.4 & 415.5 & 6.14 & -6.14 & 0 & Antibiotic \\
\hline Moxifloxacin & 2.01 & 89.5 & 401.4 & 5.87 & -5.87 & 0 & Antibiotic \\
\hline Thiolactomycin & 2.41 & 34.1 & 210.3 & 2.39 & -2.39 & 0 & Antibiotic \\
\hline Cloxacillin & 2.67 & 115.6 & 434.9 & 4.96 & -5.96 & -1 & Antibiotic \\
\hline Proflavin & 2.80 & 66.2 & 210.3 & 4.03 & -3.03 & 1 & Fluorescent probe \\
\hline Pyronin $\mathrm{Y}$ & 2.98 & 15.5 & 267.4 & 3.14 & -2.14 & 1 & Fluorescent Probe, quenched intracellular fluorescence \\
\hline Nitrocefin & 3.02 & 181.2 & 515.5 & 7.20 & -8.20 & -1 & Antibiotic \\
\hline Clarithromycin & 3.12 & 184.1 & 749.0 & 9.61 & -8.61 & 1 & Antibiotic \\
\hline Acriflavine & 3.19 & 55.9 & 224.3 & 4.06 & -3.06 & 1 & Fluorescent Probe, quenched intracellular fluorescence \\
\hline Leu-NAP & 3.29 & 56.7 & 257.4 & 4.02 & -3.02 & 1 & Intracellular conversion into a fluorescent product \\
\hline Novobiocin & 3.30 & 198.9 & 611.6 & 8.03 & -9.04 & -1 & Antibiotic \\
\hline ANS & 3.35 & 63.2 & 298.3 & 3.71 & -4.71 & -1 & Fluorescent Probe, enhanced intracellular fluorescence \\
\hline Nile red & 3.71 & 41.9 & 318.4 & 3.38 & -3.38 & 0 & Fluorescent Probe, enhanced intracellular fluorescence \\
\hline Telithromycin & 3.84 & 173.1 & 813.0 & 9.91 & -8.91 & 1 & Antibiotic \\
\hline Fluorescein & 4.13 & 76.0 & 332.3 & 4.16 & -4.16 & 0 & Fluorescent probe \\
\hline DASPEI & 4.38 & 7.12 & 253.4 & 3.45 & -2.45 & 1 & Fluorescent Probe, enhanced intracellular fluorescence \\
\hline
\end{tabular}


TABLE 1 | Continued

\begin{tabular}{|c|c|c|c|c|c|c|c|}
\hline Compound & $\log P(o / w)^{a}$ & $\operatorname{TPSA}\left(\AA^{2}\right)^{b}$ & MW (Da)c & $P C+{ }^{d}$ & PC-e & Net PC & Properties \\
\hline Hoechst H33342 & 4.47 & 74.3 & 453.6 & 6.09 & -5.09 & 1 & Fluorescent Probe, enhanced intracellular fluorescence \\
\hline NPN & 4.51 & 12.0 & 219.3 & 2.40 & -2.40 & 0 & Fluorescent Probe, enhanced intracellular fluorescence \\
\hline Rifampicin & 4.56 & 221.4 & 824.0 & 11.1 & -10.1 & 1 & Antibiotic \\
\hline Ethidium & 5.20 & 55.9 & 314.4 & 4.66 & -3.66 & 1 & Fluorescent Probe, enhanced intracellular fluorescence \\
\hline TMA-DPH & 5.39 & 0.0 & 290.4 & 4.18 & -3.18 & 1 & Fluorescent Probe, enhanced intracellular fluorescence \\
\hline Rhodamine 6G & 5.76 & 59.9 & 442.6 & 4.23 & -4.24 & 0 & Fluorescent probe \\
\hline $1,2^{\prime}$-dinaphthylamine & 5.77 & 12.0 & 269.3 & 2.70 & -2.70 & 0 & Fluorescent Probe, enhanced intracellular fluorescence \\
\hline $\mathrm{DiOC}_{2}(3)$ & 6.06 & 29.5 & 333.4 & 3.79 & -2.79 & 1 & Fluorescent Probe, enhanced intracellular fluorescence \\
\hline \multicolumn{8}{|l|}{ Other } \\
\hline Imipenem & -0.69 & 118.3 & 299.4 & 5.50 & -5.50 & 0 & Antibiotic, not a substrate \\
\hline Resorufin & 1.49 & 58.9 & 213.2 & 2.75 & -2.75 & 0 & Fluorescent probe \\
\hline Optochin & 3.75 & 46.8 & 341.5 & 4.36 & -3.37 & 1 & Quinolone \\
\hline
\end{tabular}

Descriptors were calculated for pH 7 using Molecular Operating Environment (MOE), 2013.08; Chemical Computing Group Inc., 1010 Sherbooke St. West, Suite \#910, Montreal, QC, Canada, H3A 2R7, 2014.

a Logarithm of partition coefficient between $n$-octanol and water (o/w); ${ }^{\mathrm{b}}$ Total Polar Surface Area; ${ }^{\mathrm{c}}$ Molecular Weight; ${ }^{\mathrm{d}}$ Partial positive charge; e Partial negagtive Charge; ${ }^{f}$ net Partial Charge.

gExamples for which efflux inhibition was shown are listed. Other substrates may act as inhibitors too.

Note that most antibiotics are amphiphilic compounds with hydrophobic parts that are required to partition into a membrane but that there are considerable differences with regard to the impact of efflux on activity even within classes of antibiotics (Hancock, 1997; Nikaido, 2003; Delcour, 2009; Brown et al., 2014).

An effort to make tetracyclines that are not substrates of tetracycline-specific pumps of the major facilitator superfamily (MFS), led to the development of tigecycline (Chopra, 2002). However, tigecycline was still a substrate of the RND pumps MexY, MexB, and MexD in P. aeruginosa (Dean et al., 2003; Visalli et al., 2003; Chollet et al., 2004). This observation illustrates the flexibility of RND efflux pumps but it does not mean that a given RND transporter would accept all antibiotics of the same class. The macrolides erythromycin and clarithromycin have been found to be better substrates of AcrB of E. coli and of Enterobacter aerogenes than the ketolide telithromycin (Dean et al., 2003; Visalli et al., 2003; Chollet et al., 2004). RND pumps in $P$. aeruginosa have been shown by in vitro susceptibility studies to transport substrates of the quinolone family with differential preference (Li et al., 1995; Kohler et al., 1997; Lomovskaya et al., 1999; Masuda et al., 2000a; Griffith et al., 2006; Dunham et al., 2010; Morita et al., 2015). Fluoroquinolones like levofloxacin, ciprofloxacin, norfloxacin, and others, have been reported to be most efficiently exported by MexEF-OprN and MexCD-OprJ and with less efficiency by MexAB-OprM and MexXY-OprM (or OprA). Non-fluorinated quinolones (e.g., nalidixic acid, piromidic acid, pipemidic acid, cinoxacin, oxolinic acid, and flumequine) have been shown to be preferentially transported by MexEF-OprN, less efficiently by MexCD-OprJ and MexABOprM, and least efficiently by MexXY-OprM (Kohler et al., 1997; Masuda et al., 2000b). It has been concluded from these results that fluoroquinolones with a positive charge and an electronegative fluorine atom are predominantly recognized by MexCD-OprJ, whereas quinolones without the fluorine atom are preferred substrates of MexEF-OprN. This result was in agreement with earlier work, suggesting that MexCD-OprJ expels amphiphilic substrates with a positive charge that can partition into a membrane (Masuda et al., 1996; Nikaido, 1996).

Studies on $\beta$-lactam efflux by AcrB in Salmonella enterica serovar Typhimurium have indicated that RND efflux pumps somehow recognize the hydrophobicity of a substrate (Nikaido et al., 1998; Ferreira and Kiralj, 2004). The studies showed that efflux efficiency varied with the lipophilicity of the $\beta$-lactam side chain and that molecules with more hydrophobic side chains were preferred substrates. Further work with $\beta$-lactams shed light on substrate specificity among RND pumps in $P$. aeruginosa. MexB transported a broad spectrum of $\beta$-lactams including penicillins, cephems, and carbapenems but not imipenem (Masuda et al., 2000b, 2001; Baum et al., 2009). MexD had a slightly narrower spectrum, which excluded the MexB substrates carbenicillin, sulbenicillin, ceftazidime, and moxalactam (Li et al., 1994; Masuda et al., 2000b). MexY had a yet narrower spectrum, which further excluded cefsulodin, flomoxef, and aztreonam (Masuda et al., 2000b; Hocquet et al., 2006). As carbenicillin and sulbenicillin have a negative charge that the other $\beta$-lactams in theses studies do not have, it seems that MexB accepts substrates with negative charges that MexD and MexY do not recognize. The finding that certain variants of MexD had altered substrate specificity has suggested that the recognition likely takes place via electrostatic interactions. MexD Q45K and MexD E89K with additional positively charged lysine residues have conferred resistance to the negatively charged carbenicillin, aztreonam, and ceftazidime (Mao et al., 2002). A role of charged residues in substrate recognition has been shown for AcrD too based on the fact that the binding pocket of AcrD contains an arginine residue (R625) not found in the mostly hydrophobic pocket of AcrB. Resistance to negatively charged $\beta$-lactams increased significantly when an arginine residue was introduced at the corresponding place in AcrB (I626R). Removal of a glutamic acid residue from the AcrB pocket (E673G), a residue not present at this position in $\mathrm{AcrD}$, further enhanced the protective 
effect and helped to explain the specificity of AcrD for small hydrophilic substrates such as the anionic $\beta$-lactams carbenicillin, aztreonam, and sulbenicillin (Wehmeier et al., 2009; Kobayashi et al., 2014).

Carbapenem activity was not uniformly affected by efflux pumps either. Meropenem was sensitive to overexpression of MexAB-OprM, MexCD-OprJ, or MexXY-OprM, whereas imipenem was not significantly affected (Masuda et al., 2000b; Ong et al., 2007; Riera et al., 2011; Castanheira et al., 2014). Efflux-pump expression levels in clinical isolates of $P$. aeruginosa indicated that MexAB-OprM and MexXY-OprM were most relevant for meropenem activity, followed by MexEF-OprN and with the lowest impact by MexCD-OprJ (Pai et al., 2001; Giske et al., 2005; Riera et al., 2011). A computer simulation of imipenem and meropenem transport by MexB suggested that the hydrophobicity and the flexibility of the side chains were probably responsible for efflux sensitivity (Collu et al., 2012b). The study indicated that the more rigid and hydrophobic tail of meropenem made strong interactions with the hydrophobic lining of the distal binding pocket in MexB (see Figure 1 for the structure of the transporter with two affinity sites) whereas the flexible and more hydrophilic tail of imipenem prevented this interaction (a detailed description of the extrusion pathway is given in the Section Computational Study). The computational results have further suggested that imipenem had no pronounced affinity for any particular site in MexB.
The anti-MRSA cephalosporin ceftobiprole is another $\beta$-lactam that is poorly recognized by MexB, MexD, and MexF in $P$. aeruginosa (Baum et al., 2009). Overexpression of these efflux pumps had only the marginal effect on the activity of ceftobiprole of a twofold increase of the minimum inhibitory concentration (MIC). Overexpression of MexXY caused a fourfold to eightfold increase of the MIC. The data have indicated that ceftobiprole was more efficiently transported by MexY than by MexB (Baum et al., 2009).

It should be pointed out that not all efflux substrates can be reliably detected by MIC. MIC may be a misleading indicator for efflux for any antibacterial that is effective at a concentration below the concentration required to reach maximal efflux velocity $V_{\text {max }}$. For example, AcrB had only a minor effect on the activity of cefaloridine (Nikaido et al., 1998; Mazzariol et al., 2000). Cefaloridine was later shown to be strongly effluxed by AcrB only at concentrations higher than those required to kill bacteria (Nagano and Nikaido, 2009). The discrepancy was explained by the finding that cefaloridine was effluxed with a high $V_{\max }$ but also with a strong positive cooperativity. The cefaloridine concentration required to reach half-maximal velocity $(288 \mu \mathrm{M})$ was much higher than the effective antibacterial dose (Nikaido and Normark, 1987; Nagano and Nikaido, 2009). Note that cefaloridine may not be an exception because other drugs (cephalotin, cefamandole) were also transported in a cooperative manner (Nagano and Nikaido, 2009). The situation may be
A

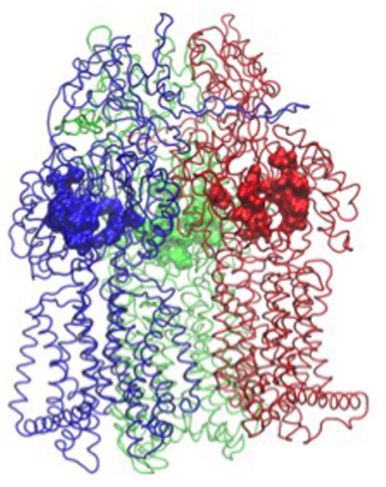

B

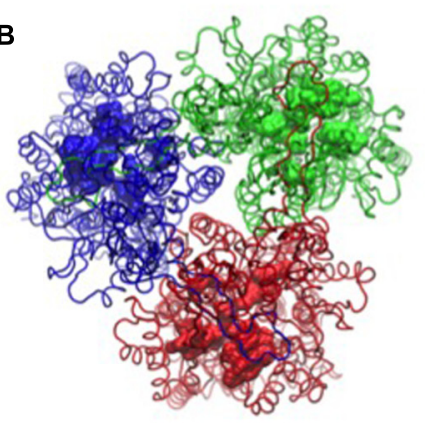

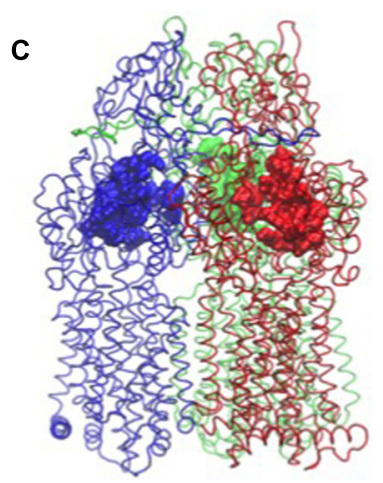
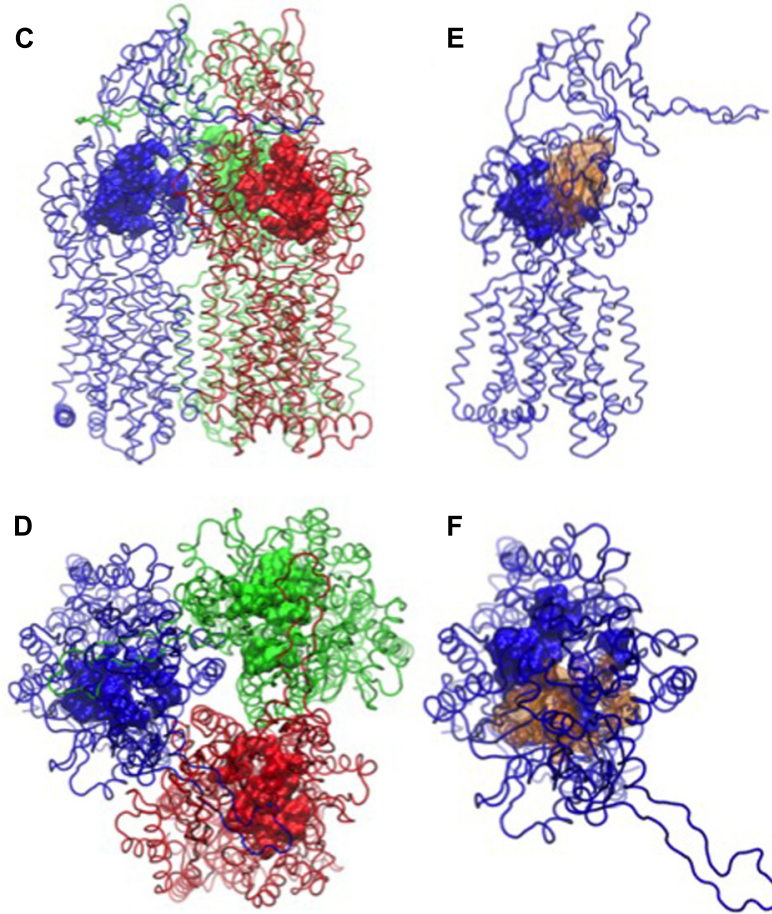

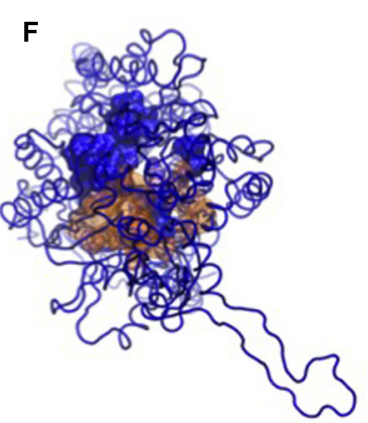

FIGURE 1 | Binding pockets of MexB [PDB code: 2V50 (Sennhauser et al., 2009)]. The monomers $L, T$, and $O$ are in tube representation and colored in blue, red, and green, respectively. In (A,B) side and top views of MexB with the distal binding pocket depicted as surface, in $(\mathbf{C}, \mathbf{D})$ the access binding pocket is highlighted as surface. In (E,F) the side and top views of $L$ monomer are shown with access and distal binding pocket colored as blue and yellow surfaces, respectively. All the atomic-level figures were rendered using Visual Molecular Dynamics (VMD; Humphrey et al., 1996). 
different in $P$. aeruginosa because its $\mathrm{OM}$ has a lower permeability for $\beta$-lactams than the OM of $E$. coli (as explained before). This lower permeability leads to higher MIC and to a stronger impact of efflux than in E. coli and thus, less discrepancy between efflux studies and MIC data may be expected.

MIC tests in the presence of the model efflux-pump inhibitor (EPI) phenylalanine-arginine $\beta$-naphthylamide $(\mathrm{PA} \beta \mathrm{N})$ are often done as a quick check for efflux effects (e.g., Mesaros et al., 2007; Pages et al., 2009; Castanheira et al., 2014). Increased antimicrobial activity (i.e., lower MIC) of an antibiotic in the presence of $\mathrm{PA} \beta \mathrm{N}$ is taken as an indication that the test compound is an efflux substrate. However, detailed studies with $\mathrm{PA} \beta \mathrm{N}$ nicely illustrated the complexity of effects that can influence whole cell assays such as MIC determinations. It has already been mentioned in the original publication of $\mathrm{PA} \beta \mathrm{N}$ that this agent does not only inhibit efflux pumps but also permeabilizes the OM of Gram-negative bacteria (Lomovskaya et al., 2001). This permeabilization property has been confirmed later in separate studies (Iino et al., 2012b; Lamers et al., 2013). Permeabilization of the OM may be mistaken as pump inhibition because facilitated entry of test compounds leads to increased intracellular levels. Pump inhibition and membrane permeabilization by $\mathrm{PA} \beta \mathrm{N}$ have been shown to be dosedependent, separable activities in E. coli because AcrB and AcrF have been specifically inhibited at low doses of $P A \beta N$, whereas membrane destabilization has been observed at higher concentrations of PA $\beta \mathrm{N}$ (Misra et al., 2015).

The protonophore carbonyl cyanide m-chlorophenylhydrazone (CCCP) is often used in a similar way as PA $\beta N$ to probe for efflux phenomena. RND pumps belong to the class of secondary transporters, which are driven by a transmembrane electrochemical potential of protons (reviewed in BorgesWalmsley et al., 2003; Poole, 2005; Dreier, 2007). CCCP indirectly inhibits secondary efflux pumps by dissipating the proton gradient across the inner membrane (Rosen and Kashket, 1978; Levy, 1992). It should be clear that whole cell assays require thorough controls to avoid confusion of pump inhibition with other effects. Susceptibility tests are useful to investigate general substrate recognition patterns whereas elucidation of detailed efflux mechanisms requires specifically designed assays.

\section{Efflux Assays}

Transport studies generally require at least two compartments and a method to measure concentration changes of a tracer substance. Entire RND efflux systems require three compartments (cytosol, periplasm, and cell exterior), which makes whole bacteria an obvious choice for efflux assays. In principle, cells can be incubated with any pump substrate and efflux can be measured by following the change of substrate concentration inside or outside of the bacteria [e.g., (Kohler et al., 1997; Pumbwe and Piddock, 2000)]. Such a generic method may require the separation of intracellular from extracellular substrate, usually achieved by filtration or centrifugation. Cell lysis may be necessary too before a versatile detection method like liquid chromatography-mass spectrometry (LC-MS) or high performance liquid chromatography (HPLC) can be applied (e.g., Schumacher et al., 2007; Cai et al., 2009). Homogeneous assays use methods that allow substrate quantification without a separation step, which simplifies applications like time-course transport studies or high-throughput tests. Fluorescence was selected as the method of choice to assess fluoroquinolone accumulation in Gram-negative bacteria based on a comparison of various methods (Mortimer and Piddock, 1991). The study showed that steady-state concentrations and times to reach the steady-state condition varied in a substrate-specific manner within the quinolone family.

Many protocols use molecules whose fluorescent properties are sensitive to their environment and change upon entry into a cell (Figures 2A,B). A prominent example is ethidium bromide (EtBr) since the quantum yield of the fluorescence increases when ethidium intercalates into DNA (LePecq and Paoletti, 1967; Mine et al., 1999; Morita et al., 2001; Li et al., 2003). Active efflux causes reduced intracellular levels of EtBr and as a consequence a decrease of fluorescence whereas inhibition of efflux pumps is recorded as a signal increase (Figure 2A). The same assay principle (Figure 2A) applies to 1-anilinonaphtalene8-sulphonate (ANS) accumulation because the fluorescence quantum yield increases when ANS binds to hydrophobic structures (e.g., proteins, membranes) in the cell. ANS is a substrate of MexD and has been used to study this transporter in P. aeruginosa (Walmsley et al., 1994; Mao et al., 2002; Kamal et al., 2013). Another example is $1,2^{\prime}$-dinaphthylamine which has been used to measure AcrB efflux in E. coli because it is a substrate of AcrB and becomes strongly fluorescent when it partitions into a phospholipid bilayer (Bohnert et al., 2011a).

A widely used fluorophore with increased intracellular fluorescence is the bis-benzamide dye Hoechst H33342 which becomes more fluorescent upon binding to DNA (Loontiens et al., 1990). H33342 was successfully used for accumulation studies with several Gram-positive bacteria, Enterobacteriaceae, and Acinetobacter baumannii (van den Berg van Saparoea et al., 2005; Garvey and Piddock, 2008; Richmond et al., 2013). Transport of $\mathrm{H} 33342$ by intact $P$. aeruginosa has been difficult to measure because of the low OM permeability for such hydrophobic dyes (Loh et al., 1984; Ocaktan et al., 1997; Germ et al., 1999). However, MexB readily transported $\mathrm{H} 33342$ when it was expressed in an E. coli host (Welch et al., 2010; Ohene-Agyei et al., 2012). This example shows that whole cell assays reflect a sum of events, which often need to be dissected to identify unambiguously substrates of a given efflux pump.

Another group of efflux probes allows efflux measurements based on a signal decrease upon intracellular accumulation (Figure 2B; e.g., Mao et al., 2002; Nakashima et al., 2013). Examples are the anthracycline cancer drug doxorubicin, whose fluorescence is quenched when the drug is internalized, Pyronin $\mathrm{Y}$, whose intracellular fluorescence is quenched because of binding to RNA (Nishino and Yamaguchi, 2002; Nakashima et al., 2013), and acriflavine, whose fluorescence intensity decreases when it is bound to DNA (Chen et al., 2002). Pyronin Y has been successfully used for transport studies with MexD in P. aeruginosa (Mao et al., 2002). 


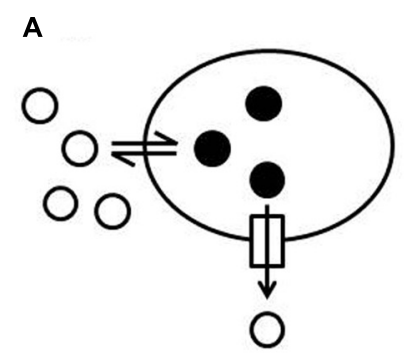

B

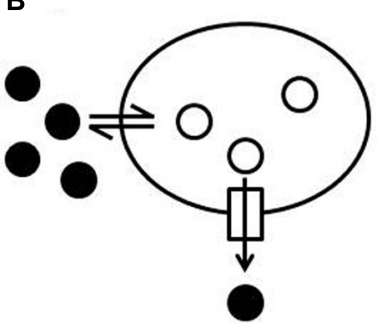

C

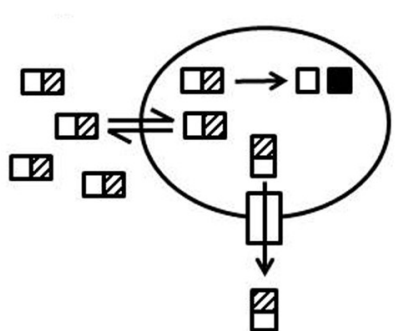

FIGURE 2 | Schematic representation of efflux assay formats. (A) Increase of fluorescence intensity upon internalization of a probe. A probe with low fluorescence intensity in the medium surrounding the cells (open circles) is taken up by cells (double arrow). The fluorescence intensity of the probe increases when the probe is internalized (black circles) and interacts with cellular structures such as DNA, proteins, or membranes. Efflux of the probe (arrow through the box to the outside) causes a decrease of the total fluorescence and efflux inhibition causes an increase of the total fluorescence. (B) Quenching of fluorescence intensity upon internalization of a probe. A fluorescent probe (black circles) is added to the medium surrounding the cells. The fluorescence intensity of the probe decreases when the probe is taken up by the cells (open circles) and interacts with cellular structures (e.g., DNA and RNA). Efflux of the probe causes an increase of the total fluorescence and efflux inhibition causes a decrease of the total fluorescence.

(C) Intracellular conversion of a probe into a fluorescent product. A non-fluorescent probe (rectangles with a hatched box) is added to the medium surrounding the cells. The probe can penetrate into the cells where it is enzymatically converted (e.g., cleaved or reduced) into a fluorescent product (black squares). The conversion rate of the intact probe into the fluorescent product depends on the intracellular concentration of the intact probe. Efflux of the intact probe slows down the production of the fluorescent product and efflux inhibition increases the rate of fluorescent product generation.
Intracellular conversion of a substrate into a traceable entity is another elegant way to develop homogeneous efflux assays (Figure 2C). The non-fluorescent, blue-colored resazurin (sold under the trade name Alamar Blue ${ }^{\circledR}$ ) is reduced to the fluorescent, pink-colored resorufin in live cells by oxidoreductases (Gonzalez and Tarloff, 2001; Rampersad, 2012). The conversion is an indicator mainly for the presence of NADH but also for NADPH and FADH and is routinely used to detect living cells. Resazurin can be used to monitor bacterial growth because conversion rates depend on cell density. However, the method does not work equally well with all microorganisms and is not recommended for $P$. aeruginosa. It was shown that resazurin is a substrate of $\mathrm{MexB}$ in $P$. aeruginosa and of AcrB in $E$. coli and it was suggested that efflux pump activity in $P$. aeruginosa may account for the difficulties encountered with resazurin (Vidal-Aroca et al., 2009). Vidal-Aroca et al. (2009) showed that under conditions where Pseudomonas do not grow (i.e., in phosphate buffered saline), resazurin can be used to measure efflux pump activity. The assay was useful to identify mefloquine as an inhibitor of MexB and AcrB and to show that optochin, another quinoline derivative, was not an inhibitor of these pumps.

The non-fluorescent alanine- $\beta$-naphthylamide (Ala-NAP) and leucine- $\beta$-naphthylamide (Leu-NAP) are enzymatically converted to the highly fluorescent $\beta$-naphthylamide by cellular aminopeptidases (Marks et al., 1981; Butler et al., 1993). Assays for RND efflux were developed with Ala-NAP and Leu-NAP and used for the development of peptidomimetic EPI including the model EPI PA $\beta N$ (Lomovskaya et al., 2001; Mao et al., 2002).

Fluorescein-di- $\beta$-D-galactopyranoside (FDG) is hydrolyzed in the cytoplasm of $E$. coli by $\beta$-galacotsidase to produce fluorescein (Russo-Marie et al., 1993; Fieldler and Hinz, 1994; Yang and $\mathrm{Hu}, 2004)$. Both FDG and fluorescein were shown to be effluxpump substrates and could be used to develop transport assays with E. coli cells expressing the pseudomonal MexAB-OprM and MexXY-OprM efflux systems (Matsumoto et al., 2011). Intracellular conversion of FDG into fluorescein could even be followed in single-cell mode with a microfluidic device using E. coli (Matsumoto et al., 2011; Iino et al., 2012b). FDG cannot be hydrolyzed by $P$. aeruginosa but FDG was useful to study MexB and MexY when they were expressed in an E. coli host (Iino et al., 2013). Another approach made use of femtoliter droplet arrays to monitor FDG based efflux in single E. coli cells (Iino et al., 2012a, 2013).

The following paragraphs describe fluorescent dyes, which were used as probes for membrane integrity or membrane potential and were later found to be substrates of efflux pumps. The physicochemical properties of these fluorescent dyes provide information on substrate recognition by efflux pumps irrespective of the original use of the probe.

The lipophilic membrane partitioning dye $N$-phenyl-1naphthylamine (NPN) is useful as a probe for membrane integrity because the amount of NPN that can partition into the phospholipid membrane and hence the fluorescence signal increases when the structure of the OM is disturbed (Loh et al., 1984; Vaara, 1992; Lomovskaya et al., 2001). NPN was shown to be a substrate of AcrB in E. coli and of MexB in P. aeruginosa (Sedgwick and Bragg, 1996; Ocaktan et al., 1997; Lomovskaya et al., 2001; Murakami et al., 2004; Cai et al., 2009).

Fluorescent potentiometric probes such as the anionic oxonols and the cationic carbocyanines and rhodamines are useful to measure changes of bacterial membrane potentials (Shapiro, 2000). The overall fluorescence signal of these so called slow-response dyes depends on the transmembrane distribution, which is sensitive to changes of the electrochemical potential across the membrane. Rhodamine measurements in $P$. aeruginosa were sensitive to the expression of the RND pumps MexAB-OprM, MexCD-OprJ, and MexHI-OpmD (Morita et al., 
2001; Sekiya et al., 2003). The physicochemical features of these fluorophores, required to partition into a membrane, are apparently recognized by RND efflux-pumps. Indeed, efflux assays were reported in various systems with dyes like the uncharged NPN (MexB of P. aeruginosa, AcrB of E. coli) or the cationic 1-(4-trimethylammoniumphenyl)-6-phenyl-1,3,5hexatriene (TMA-DPH, MexB of $P$. aeruginosa) and 2-(4dimethylamino)styryl- $N$-ethylpyridinium iodide (DASPEI, AcrB of E. coli, MexB of P. aeruginosa), (Sedgwick and Bragg, 1996; Ocaktan et al., 1997; Lomovskaya et al., 2001; Murakami et al., 2004; Cai et al., 2009). The properties of the dyes that have been identified as substrates of MexB are listed in Table $\mathbf{1}$.

Substrate export can be directly followed from pre-loaded cells. Alternatively, pump activity can be deduced from intracellular accumulation of externally added substrates, which requires less preparation but reflects efflux in an indirect manner only and includes the hurdle of cellular uptake. Direct efflux measurements require pre-loading of cells with substrate. This extra complication pays off because efflux-related effects can be distinguished from permeation phenomena. The lipophilic dye Nile Red is basically non-fluorescent in aqueous solutions but becomes fluorescent in intracellular, non-polar environments (Greenspan and Fowler, 1985). Nile Red was used for efflux measurements of AcrAB/TolC in E. coli (Greenspan and Fowler, 1985; Bohnert et al., 2010, 2011b). When E. coli cells were concomitantly loaded with substrate and inhibitor for competition studies, the strongest inhibition was seen with doxorubicin and minocycline, comparable to the effect of PA $\beta N$. Many other antibiotics caused slower Nile Red efflux, which is indicative of competitive transport. These results provided further evidence for specific substrate-pump interactions and corroborated the notion of substrate-specific inhibitor efficacy. Adaptation of the Nile Red assay for the use with $P$. aeruginosa would most likely not be straight forward because Nile Red was shown to stain extracelluar rhamnolipids made by $P$. aeruginosa (Morris et al., 2011). Strains with deletions of rhamnolipid synthesis genes (rhlAB) may be helpful even if the swarming motility patterns influenced by rhamnolipids would be changed (Deziel et al., 2003; Caiazza et al., 2005). However, pre-loading of $P$. aeruginosa has been achieved with TMA-DPH (Ocaktan et al., 1997). Several other dyes (doxorubicin, rhodamin for YhiUV, NPN, 1,2'-dinaphthylamine and DASPEI for AcrB) have been used to load E. coli for efflux assays (Lomovskaya et al., 2001; Nishino and Yamaguchi, 2002; Bohnert et al., 2011a).

Quantitative measurement of substrate transport is crucial for the characterization of efflux pumps. Kinetic analysis of EtBr transport in E. coli has shown that uptake rates increased and efflux rates decreased when AcrB was deleted (Xu et al., 2003; Viveiros et al., 2008; Paixao et al., 2009). EtBr transport by MexB has been shown in whole $P$. aeruginosa and an impressive turnover rate of $500 \mathrm{~s}^{-1}$ has been determined for $\mathrm{MexB}$ in $\mathrm{EtBr}$ efflux assays when the number of pumps was estimated by immunoblotting methods (Ocaktan et al., 1997; Narita et al., 2003). A link of MexCD-OprJ expression with EtBr efflux from $P$. aeruginosa has been demonstrated by Morita et al. (2001, 2003) when they showed that significant EtBr efflux was only measurable after induction of MexCD-OprJ. MexCD-OprJ expression was inducible with tetraphenyl phosphonium, EtBr, rhodamine 6G, acriflavine, benzalkonium chloride, and chlorhexidine gluconate but not with various antibiotics including substrates of MexD (i.e., norfloxacin, tetracycline, chloramphenicol, streptomycin, or erythromycin).

A thorough quantitative analysis of efflux was achieved with $\beta$-lactams and intact $E$. coli cells (Nagano and Nikaido, 2009). The method relies on the hydrolysis of $\beta$-lactams by periplasmatic $\beta$-lactamases and would probably not be directly applicable to $P$. aeruginosa because of the about 10-100 times lower permeability of the OM for $\beta$-lactams (Angus et al., 1982; Yoshimura and Nikaido, 1982; Parr et al., 1987). Under these circumstances it would be very difficult to saturate the efflux pumps and the method may be restricted to a few suitable $\beta$-lactams. Hydrolysis rates of $\beta$-lactams were measured in intact cells to calculate the periplasmic antibiotic concentration based on known kinetic parameters of $\beta$-lactamases. Maximal velocity and dose-response curves depended on the nature of the substrate. Positive cooperativity was detected with cefamandole, cephalotin, and cefaloridin but not with nitrocefin (Nagano and Nikaido, 2009). Cooperativity agrees with the current model of the functional complex of AcrAB-TolC where multiple substrate binding sites reside within three AcrB monomers that adopt different structural conformations in a concerted way during substrate transport (Seeger et al., 2006; Murakami et al., 2006; Sennhauser et al., 2007). Nitrocefin transport was the slowest in the series, which could be explained by the rather strong binding of nitrocefin with its two aromatic rings to AcrB $\left(K_{\mathrm{m}}\right.$ of $\left.5 \mu \mathrm{M}\right)$. No significant efflux could be measured with cefazolin, which has two hydrophilic substituents. Previous work has shown that MIC of cloxacilin, which has a lipophilic side, chain dropped from 256 to $2 \mathrm{mg} / \mathrm{L}$ in $S$. enterica serovar Typhimurium and from 512 to $2 \mathrm{mg} / \mathrm{L}$ in $E$. coli upon inactivation of AcrB whereas no change was observed for the MIC of the hydrophilic cefazolin (Nikaido et al., 1998; Mazzariol et al., 2000). MIC values and efflux studies strongly suggested that AcrB from E. coli and S. enterica serovar Typhimurium have a preference for hydrophobic $\beta$-lactams.

Specific mode-of-action studies ask for less complex assay systems than entire bacterial cells. The activity of different bacterial membrane transporters could be studied with membrane vesicles. Vesicles proved to be very valuable tools for the study of tetracycline-specific pumps (e.g., Yamaguchi et al., 1990). Tetracycline-specific pumps belong to the MFS class of transporters spanning a single cell membrane (Chopra and Roberts, 2001), whereas RND pumps are multi-subunit complexes made of pump subunits (e.g., MexB), an OM channel (e.g., OprM), and an adaptor subunit (e.g., MexA). The pump is formed by three monomers placed in the inner membrane and protrudes into the periplasm (Murakami et al., 2002, 2006; Seeger et al., 2006; Sennhauser et al., 2007, 2009). The OM channel, formed by three monomers, passes the OM linking the periplasmic space with the outside of the cell (Koronakis et al., 2000; Akama et al., 2004a; Phan et al., 2010). The third subunit is an adaptor protein that is required for the assembly of the functional complex which spans the inner membrane, the periplasmic space, and the OM. The stoichiometry of the 
subunits (MexB:MexA:OprM or AcrB:AcrA:TolC) is currently discussed with evidence for 3:6:3 (Du et al., 2014, 2015; Kim et al., 2015) or for 3:3:3 (Symmons et al., 2009; Trepout et al., 2010). The models are based on electron microscopy data at resolutions around $20 \AA$ and on docking calculations. A higher-resolution crystal structure of the assembled efflux systems will likely provide a clear-cut response to the controversy regarding the stoichiometry and the construction of the tripartite complex.

The isolated pump subunits AcrB and AcrD from E. coli and MexB from $P$. aeruginosa were reconstituted in vesicles (Zgurskaya and Nikaido, 1999; Aires and Nikaido, 2005; Verchère et al., 2014, 2015) but reconstitution of a functional tripartite RND pump complex for in vitro transport studies is a truly difficult task that has been achieved only recently (NtsogoEnguene et al., 2015; Verchère et al., 2015). Competition studies with AcrB-containing vesicles have indicated that the bile salts taurocholate and glycocholate inhibited transport of a fluorescent phospholipid more efficiently than erythromycin or cloxacillin did (Zgurskaya and Nikaido, 1999). Chloramphenicol was identified in the same study as a substrate that did not inhibit phospholipid efflux. These results suggested that known AcrB substrates were transported with different efficiency reflecting specific substrate recognition patterns. Functional MexAB-OprM complexes have been assembled in vitro in a liposome system combining proteoliposomes with OprM and proteoliposomes with MexAB in a way that MexAB-OprM was able to transport $\mathrm{EtBr}$ driven by a proton gradient (NtsogoEnguene et al., 2015). A related approach used vesicles with MexB and bacteriorhodopsin, a light activated proton pump from Halobacterium salinarium (Verchère et al., 2014). Light activation caused bacteriorhodopsin to build up a proton gradient as the energy source for MexB. A change of $\mathrm{pH}$ was then taken as indication for substrate transport. The system confirmed that $\mathrm{H} 33342$ is a substrate of MexB and suggested that MexA was required for proton-coupled transport.

\section{Efflux Inhibitors}

Many chemically diverse EPI have been reported and their selectivity for pumps can provide insight into specific inhibitorpump interactions (reviewed in Zechini and Versace, 2009; Van Bambeke et al., 2010). Inhibitors have been derived from natural products, antibiotics, drugs originating from other therapeutic areas or have been newly developed. PA $\beta \mathrm{N}$ and more advanced peptidomimetics of the same series are among the best studied examples (Lomovskaya et al., 2001; Mao et al., 2001; Renau and Lemoine, 2001; Renau et al., 2002; Watkins et al., 2003; Kriengkauykiat et al., 2005). Selectivity for pumps indicated specific inhibitor-pump interactions. For example, PA $\beta N$ was broadly active against MexAB-OprM, MexEF-OprN, MexCD-OprJ, and MexXY-OprM in P. aeruginosa as well as against AcrAB-TolC in several species of the Enterobacteriaceae family while the pyridopyrimidine efflux inhibitor D13-9001 had a much narrower spectrum with specificity for AcrB and MexB (Nakashima et al., 2013). PA $\beta N$ has been shown to have synergistic activity with levofloxacin against wild-type
$P$. aeruginosa or against $P$. aeruginosa strains overexpressing any of MexAB-OprM, MexCD-OprJ, MexEF-OprM, or MexXY (Lomovskaya et al., 2001). On the other hand, PAßN had no significant effect on carbenicillin or $\mathrm{EtBr}$, which are substrates of MexB too, suggesting multiple binding sites for different substrates. An antagonistic effect of PA $\beta \mathrm{N}$ with aminoglycosides has been observed in the presence of MexXY-OprM, which was explained by the induction of this efflux pump by PABN (Mao et al., 2001). PA $\beta \mathrm{N}$ was shown to be an efflux substrate likely to act by competition with other substrates for transport (Pages et al., 2005; Lomovskaya and Bostian, 2006; Mahamoud et al., 2007). D13-9001 was hardly transported but was shown to inhibit efflux by high affinity binding to a specific site (Nakashima et al., 2013). Many substances cannot be unambiguously classified as substrates or as inhibitors because there seems to be a gradual transition of properties and many molecules have both characteristics. Minocycline is a substrate of several RND pumps such as AcrB from E. coli, Proteus mirabilis, Morganella morganii, MexB, MexD, and MexY in P. aeruginosa, or AdeB and AdeJ in A. baumannii (Dean et al., 2003; Visalli et al., 2003; Hirata et al., 2004; Ruzin et al., 2005; Damier-Piolle et al., 2008) but it also acted as an inhibitor of nitrocefin efflux by AcrB (Takatsuka et al., 2010). Trimethoprim, an antibiotic that has been shown to be transported by several RND efflux pumps (i.e., MexB, MexD, and MexF in P. aeruginosa, AdeB, AdeJ, AdeG in A. baumannii, and BpeF in Burckholderia pseudomallei), inhibited the efflux of $\mathrm{H} 33342$ by AcrB in S. enterica serovar Typhimurium (Kohler et al., 1996; Maseda et al., 2000; Magnet et al., 2001; Coyne et al., 2010; Piddock et al., 2010; Amin et al., 2013; Podnecky et al., 2013). In fact, antibiotics could be modified to become efflux inhibitors as described in the case of fluoroquinolones, tetracyclines, or aminoglycosides (reviewed in Van Bambeke et al., 2010). In general, EPI have aromatic moieties and contain ionizable groups, which are structural features that are reminiscent of substrate characteristics deduced from antibiotic susceptibility tests (Poole, 2004, 2005).

Transport measurements are useful to identify molecules that interact with efflux pumps but investigation of interactions at a molecular level and precise mode-of-action studies require more refined methods.

\section{Binding Studies and Crystal Structures}

Binding of fluorescent substrates to purified AcrB in detergent solution could be measured by fluorescence polarization (Su and $\mathrm{Yu}, 2007)$. AcrB bound rhodamine $6 \mathrm{G}$, ethidium, and proflavin, with similar strength ( $K_{\mathrm{D}}$ of $5.5,8.7$, and $14.5 \mu \mathrm{M}$, respectively). Binding of ciprofloxacin was significantly weaker with a $K_{\mathrm{D}}$ of $74.1 \mu \mathrm{M}$. Competition studies have indicated that different binding sites may exist for different antibiotics. Experiments with purified AcrB which was immobilized to a surface, yielded $K_{\mathrm{D}}$ values of $530 \mu \mathrm{M}$ for novobiocin and of $110 \mu \mathrm{M}$ for the EPI MC-207,110 (PAßN; Tikhonova et al., 2011). Binding studies confirmed specific interaction of substrates or inhibitors with the pump subunit and revealed substrate-specific variation 
of binding strength (Vargiu and Nikaido, 2012; Vargiu et al., 2014).

Many molecules have been described to be efflux substrates or inhibitors but only a small subset thereof could be crystallized in complex with RND pumps (Ruggerone et al., 2013a). Nevertheless, X-ray structures have provided detailed information on substrate-pump interactions (Murakami et al., 2006; Seeger et al., 2006; Sennhauser et al., 2007, 2009; Nakashima et al., 2011; Eicher et al., 2012). A detailed description of the structures is provided in the next section where computational simulation approaches are discussed. The porter domain of the inner membrane transporter protrudes into the periplasmic space (Figure 1) and mediates substrate specificity as predicted from genetic studies with AcrB, AcrD, MexB, MexD and MexY (Nikaido, 2011). Gene segments coding for the periplasmatic loops of the pump subunit were swapped between AcrB and AcrD of E. coli, between MexB and MexY of P. aeruginosa, and between AcrB of E. coli and MexB of P. aeruginosa (Elkins and Nikaido, 2002; Tikhonova et al., 2002; Eda et al., 2003). Altered substrate specificities of the new constructs suggested that substrate recognition was predominantly determined by the periplasmic region of the pump. Site directed mutagenesis in $P$. aeruginosa confirmed substrate recognition sites in the periplasmatic loops of MexB and MexD (Mao et al., 2002; Middlemiss and Poole, 2004; Wehmeier et al., 2009). Q34K, E89K, and N67K mutations in MexD have led to the transport of negatively charged $\beta$-lactams that are not recognized by the wild type transporter (as discussed in the Section Impact of Efflux on Antibiotic Activity; Mao et al., 2002). A direct substrate contact for macrolide recognition was proposed for an asparagine residue in a phenylalanine-rich distal binding pocket in MexB (Wehmeier et al., 2009).

The high molecular weight substrates rifampicin, erythromycin, and doxorubicin dimers bound to an access (or proximal) binding pocket (AP) shown in Figures 1A,B, which is located close to the protein/periplasm interface (Nakashima et al., 2011; Eicher et al., 2012). The low molecular weight substrates minocycline, dodecyl- $\alpha$-D-maltoside and doxorubicin have been found in a distal (or deep) binding pocket (DP) displayed in Figures 1C,D. AP and DP have also been proposed as two successive locations visited by a substrate during the translocation cycle. The two pockets are separated by a flexible loop (G-loop or switch loop) with two phenylalanine residues (Nakashima et al., 2011; Eicher et al., 2012). Substrate recognition is governed by a phenylalanine-rich hydrophobic region in the distal pocket. Minocycline, rifampicin, and erythromycin, made direct contact with phenylalanine residues (Murakami et al., 2006; Nakashima et al., 2011; Eicher et al., 2012). The MexB-specific pyridopyrimidine efflux inhibitor D13-9001 bound to a narrow pit in the phenylalanine cluster of the distal pocket making $\pi-\pi$-stacking interactions (Nakashima et al., 2013). The hydrophilic part of the inhibitor interacted with hydrophilic and ionic residues close to the binding site of minocycline and doxorubicin (Nakashima et al., 2013). Binding to MexY was sterically hindered by a tryptophan in agreement with the phenotypic specificity for MexB over MexY (Nakayama et al., 2003; Yoshida et al., 2007). The binding pocket of AcrD contains several oxygens in contrast to the mostly hydrophobic pocket of AcrB, which could explain the specificity of AcrD for small basic, hydrophilic substrates such as the anionic $\beta$-lactams carbenicillin, aztreonam, and sulbenicillin (Kobayashi et al., 2014). Domain swapping experiments have shown that the specificity of MexB for anionic $\beta$-lactams, which are not recognized by MexY, is determined by the periplasmatic domain of the pump subunit (Eda et al., 2003). Although, the complexity of substrate recognition by RND efflux systems has hindered the establishment of a straightforward criterion for the definition of good and poor substrates, it is possible that MexY, as shown for MexD and described before (Mao et al., 2002), does not have the required binding places (i.e., positively charged amino acid residues) in the substrate binding sites to accept negatively charged $\beta$-lactams. It cannot be excluded that other, subtle differences in the RND transporter-compound interaction patterns might affect the recognition and the transport at different extrusion stages and positions in the transporters (see Figure 1 where the different affinity sites are shown). Examples for such subtle but crucial interactions are imipenem and meropenem interacting with MexB (see Section Computational Study).

Mutagenesis of D133 in MexY of P. aeruginosa (D113A and D133S) has compromised resistance to several aminoglycosides but not to spectinomycin (Lau et al., 2014). The mutation of Y613A in the same protein affected resistance to aminoglycosides but not to erythromycin. This finding supported the view that substrate recognition is governed by binding to specific sites on the extrusion pathway because D133 and Y613 are located in a region of MexY that structurally corresponds to the proximal binding pocket of AcrB.

The experimental data discussed in this review, have provided evidence that hydrophobic moieties are a key property recognized by RND pumps and that hydrophilic parts of a substrate fit into mainly hydrophilic cavities adjacent to a phenylalanine-rich, hydrophobic pocket. Substrates of MexB, which are discussed in this review, are listed in Table 1.

\section{Computational Study}

Simulations have reached an impressive maturity as reflected by the increasing number of publications in the field. In particular, a range of simulation techniques have been developed and successfully applied to describe diverse biological systems. Highly precise quantum mechanical techniques (for example see the reviews of Dal Peraro et al., 2007; Zhou et al., 2010; Sgrignani and Magistrato, 2013; Steinbrecher and Elstner, 2013), the classical force-field-based approaches (Kollman et al., 2000), hybrid methods combining appropriate quantum mechanical and classical descriptions (Senn and Thiel, 2009; Steinbrecher et al., 2012) and multi-scale and statistical mechanical methodologies (Kamerlin et al., 2011; Karplus, 2014) are examples of such computational methods.

Although they have been successfully applied to several systems and used to tackle biology-inspired questions, these in silico techniques have been barely used to investigate bacterial 
efflux systems, in particular members of the RND family. Probably hampered by the lack of crystal structures of the whole systems (the single components have been crystallized) and by the complexity of the machineries (they are tripartite systems), computational studies have addressed RND efflux systems only recently (Collu and Cascella, 2013; Ruggerone et al., 2013a,b). Classical molecular dynamics (MD) simulations represent a particularly promising technique to cast a glimpse on the dynamic behavior of a protein and its immediate micro-environment. MD simulations offer insight into molecular behavior at a temporal and structural accuracy not reached by any other experimental technique today. Continuous improvement of the techniques is pushing the limits of the simulation processes toward longer simulations and thus to a description of increasingly larger biological systems. Extension of the simulation times has improved the quality of the predictions and allowed more robust evaluation of key features such as free energy of binding, interaction patterns, solvent interactions, and interaction lifetimes. Surely, limitations are still present due to the size of many systems and the length of processes of interest, but specific techniques to overcome these drawbacks are under continuous development. As an example of methods used to bridge the time gap between simulated and real processes involving RND efflux systems, we can quote targeted MD simulations (Schlitter et al., 1993), which allows induction of conformational changes between two known states, and metadynamics (Laio et al., 2005; Laio and Gervasio, 2008; Biarnés et al., 2011), which is used to simulate rare events and provides free energy profiles associated with possible processes. Advantages and drawbacks of these biased techniques have been extensively discussed in several publications, to which the interested reader is referred (e.g., Chipot, 2008; Laio and Gervasio, 2008; Markwick and McCammon, 2011; Ovchinnikov and Karplus, 2012 and references therein).

Resistance-Nodulation-Cell Division efflux systems of Gram-negative bacteria form tripartite complexes. The innermembrane RND transporter and a membrane fusion (adaptor) protein (MFP) connect to a channel that traverses the OM called the outer membrane factor or channel (OMF; Ma et al., 1993; Thanassi et al., 1997; Zgurskaya and Nikaido, 1999; Murakami et al., 2002). Some OMFs such as TolC in E. coli are highly versatile and involved in the efflux of both antibiotics and proteins as part of different efflux systems (Akama et al., 2004a; Koronakis et al., 2004; Pietras et al., 2008; Phan et al., 2010; Hinchliffe et al., 2013; Krishnamoorthy et al., 2013). The MFP is suggested to stabilize the assembly of the pump, to contribute to the transfer of efflux-coupled conformational transitions from the RND transporter to the OMF and to affect substrate recognition (Ma et al., 1993; Akama et al., 2004b; Mikolosko et al., 2006). X-ray structures of the individual components of AcrAB-TolC and MexAB-OprM (the two main RND efflux systems of $E$. coli and $P$. aeruginosa, respectively) have been solved by X-ray crystallography, and also computational studies addressing structural and dynamical aspects of these components have been reported in the literature (Vaccaro et al., 2006, 2008; Schulz and Kleinekathöfer, 2009; Schulz et al., 2010, 2011, 2015; Fischer and Kandt, 2011, 2012; Vargiu et al., 2011; Raunest and
Kandt, 2012; Wang et al., 2012; Eicher et al., 2014; Fischer et al., 2014; Blair et al., 2015a). Still open are crucial questions about structure and stoichiometry of the functional assembly (Bavro et al., 2008; Krishnamoorthy et al., 2008; Misra and Bavro, 2009; Symmons et al., 2009; Tikhonova et al., 2011; Xu et al., 2011; Ferrandez et al., 2012; Hinchliffe et al., 2013; Du et al., 2014), and also computational studies on the assembly are still in their infancy and limited to static aspects, not least because of the size and complexity of the whole systems (Symmons et al., 2009; Phillips and Gnanakaran, 2015).

The RND inner-membrane transporter subunit is integral to the function of the system. The transporter subunit of the RNDtype tripartite complex functions as a proton/drug homotrimeric antiporter and is key for energy transduction and substrate specificity of the entire three-component complex (Murakami, 2008). Structural data have mainly been collected for AcrB, of E. coli, and to a lesser extent for MexB, the homolog of AcrB in $P$. aeruginosa (for a recent review, see Ruggerone et al., 2013a). According to crystallographic results, the shape of the protein resembles that of a jellyfish. Viewed orthogonally to the membrane plane, each protomer elongates for $\sim 120 \AA$, comprising a TM region of $\sim 50 \AA$ composed of $12 \alpha$-helices (TM1 to TM12), and a periplasmic headpiece of about $70 \AA$, the latter being divided into a pore (porter) region and an upper region close to the lower part of the OMF (Murakami, 2008; Eicher et al., 2009).

After the first symmetric structure was solved (Murakami et al., 2002) other crystal structures of AcrB revealed asymmetric conformations of the three monomers in the trimer (Murakami et al., 2006; Seeger et al., 2006; Sennhauser et al., 2007). The three conformations were proposed to represent three consecutive states in a three-step peristaltic mechanism of the substrate translocation [called functional rotation (Murakami et al., 2006) or peristaltic motion (Seeger et al., 2006)]. The postulated functional rotation starts with recognition of substrate at a low affinity site on the L monomer, namely the AP. Global conformational transition converts the $\mathrm{L}$ to a $\mathrm{T}$ conformation, accompanied by tight binding of the substrate in a designated high-affinity binding pocket, i.e., the DP. Successively, a second peristaltic motion leads to a switch from the $\mathrm{T}$ to an $\mathrm{O}$ conformation, resulting in the release of the substrate toward the OMF. After substrate release, the $\mathrm{O}$ conformation relaxes back to the $\mathrm{L}$ conformation restarting the cyclic event. The conversion from the $\mathrm{T}$ to the $\mathrm{O}$ conformation is suggested to be the major energy-requiring step and should be accompanied by proton binding at the proton translocation site in the TM region. Proton release may occur during conversion from $\mathrm{O}$ to L. Computational studies based on targeted MD simulations supported this mechanism and the zipper-like closure of the DP (Schulz et al., 2010).

A $3.0 \AA$ crystal structure of MexB showed the same overall fold as its close homolog AcrB did (Sennhauser et al., 2009). The three monomers constituting MexB assumed an asymmetric conformation supporting the general transport model for this family of multi-drug transporters derived from the AcrB structures (Murakami et al., 2006; Seeger et al., 2006; Sennhauser et al., 2007). However, the conformation of monomer L in MexB 
showed significant differences at the periplasmic portal to AcrB. In particular, no access to the binding cavity was observed in this subunit. A clear rationale for this structural difference is missing to date. Sennhauser et al. (2009) proposed, among other things, that the differences might be attributed to the L monomer being trapped in an intermediate conformational state between the extrusion and the binding of the substrates. Alternatively, specific resting states of MexB and of AcrB may account for the observed differences. Recently, Nakashima et al. (2013) solved the structure of MexB in complex with a pyridopyrimidine derivative. This work provided the first structural information on MexB-inhibitor interactions. The authors also crystallized free MexB and found a structure largely similar to that described by Sennhauser et al. (2009). The binding geometry of the pyridopyrimidine derivative to AcrB was also resolved, and showed relevant variations in the conformation of the ligand. These findings highlighted that subtle differences in the mechanisms of drug binding and translocation are relevant for the two pumps.

Several key features of the MexB structure, in particular the AP and DP sites, could be mapped onto the structure of AcrB (Murakami et al., 2006; Nakashima et al., 2011; Eicher et al., 2012). The first and until now the only computational study of antibiotic-MexB interactions was based on these data (Collu et al., 2012b). Collu et al. (2012b) investigated the behavior of two carbapenems, meropenem and imipenem, in the AP and the DP. The two structurally related compounds are differently affected by the RND efflux pumps. The activity of meropenem is significantly reduced by MexAB-OprM efflux whereas the activity of imipenem is not (Masuda and Ohya, 1992; Pai et al., 2001; Pournaras et al., 2005; Walsh and Amyes, 2007). The different sensitivities to RND efflux make imipenem and meropenem attractive candidates for a comparative study of carbapenemefflux-pump interactions.

Lacking a crystal structure of $\mathrm{MexB}$ in complex with the two molecules, the starting configurations for all-atom MD simulations were extracted from docking runs with the ATTRACT package (May and Zacharias, 2008; May et al., 2008; de Vries and Zacharias, 2012). The systems obtained after solvation and equilibration were simulated for 50 ns. A stronger preference of meropenem for the DP than for the AP resulted from the simulations (binding free energies of -8.1 and $2.4 \mathrm{kcal} \mathrm{mol}^{-1}$, respectively). Imipenem had nearly the same low affinity for both pockets ( 0.6 and $0.4 \mathrm{kcal} \mathrm{mol}^{-1}$, respectively). This result agreed with microbiological data showing a fourfold to eightfold increase of the MIC of meropenem but no significant change of the imipenem MIC upon overexpression of MexB in P. aeruginosa (Eguchi et al., 2007; Ong et al., 2007; Livermore, 2009; Riera et al., 2011). The contacts between meropenem and the DP extracted from the trajectories were qualitatively consistent with the recently determined structure of a MexBinhibitor complex (Nakashima et al., 2013), suggesting a reliable prediction of the binding structures by the computational protocol of Collu et al. (2012b).

The AP is probably the first internal site to be occupied by compounds during the binding process. At the AP, imipenem and meropenem pointed their $\beta$-lactam rings toward the periplasmic region and the entrance to the DP, respectively. These could be considered as fingerprints of the different behavior of the two compounds. Meropenem tended to move toward the DP, following the plausible extrusion route, while imipenem had a propensity to reach regions close to the periplasm. Interactions with solvent molecules can be extracted and quantified from the trajectories as a further interesting detail in support of this picture (Sterpone et al., 2001; Collu et al., 2012a). Collu et al. (2012a) found that imipenem, unlike meropenem, formed long-lifetime interactions with water molecules inside of MexB.

The docking poses of two compounds in the DP were similar but the 50 ns-long simulations led to different equilibrium binding modes. Meropenem moved in the DP of MexB toward the external channel, assuming a location suitable for extrusion. Imipenem slid away from the entrance to the channel connecting the DP to the extrusion mouth and into a position similar to that assumed by doxorubicin in MD simulations of mutated AcrB F610A (Vargiu et al., 2011). Indeed, Vargiu et al. (2011) found that doxorubicin moved deeper into the DP of a F610A mutant and was not extruded by the induced functional rotation. This was in accordance with reduced doxorubicin MIC (i.e., increased activity) against the F610A variant of AcrB (Bohnert et al., 2008).

The calculated pump interaction patterns have been associated with the different physicochemical properties of the ligand molecules. With its bulky and hydrophobic tail, meropenem established a strong interaction pattern to the aromatichydrophobic environment of the DP. Conversely, the more flexible and hydrophilic tail of imipenem had a lower affinity for the DP. Solvent interactions played a major role in the different transport properties of the two carbapenems as well. In fact, the compounds remained highly solvated at all explored binding sites (Collu et al., 2012b). Nonetheless, the water dynamics around meropenem were significantly different in the DP than in the bulk solvent. On the other hand, imipenem showed the same solvent interactions in the DP as in the bulk solvent.

\section{Paths for Substrate Entry}

Substrate uptake by RND pumps is still largely unexplored and to our knowledge, only one computational study has addressed this issue (Yao et al., 2013). The entry of substrates into AcrB was studied by a combination of in silico tools and sitedirected mutagenesis. The study indicated that uptake pathways of minocycline, acriflavine, and novobiocin differed significantly. Novobiocin is the largest and acriflavin the smallest among the three compounds while minocycline is more hydrophilic than the nearly equally hydrophobic novobiocin and acriflavin. All three molecules are typical substrates of AcrB but they vary in molecular size and hydrophobicity. A ligand-dependent drug uptake mechanism was proposed based on the analysis of the free energy associated with drug movement along AcrB tunnels. Strongly hydrophobic and lipophilic drugs of similar size were preferentially taken up via the vestibule path (the entrance of the vestibule path is shown in Figures 3A,B). This path starts close to the membrane surface at a region between two protomers and goes via the AP to the DP. Other drugs were translocated 


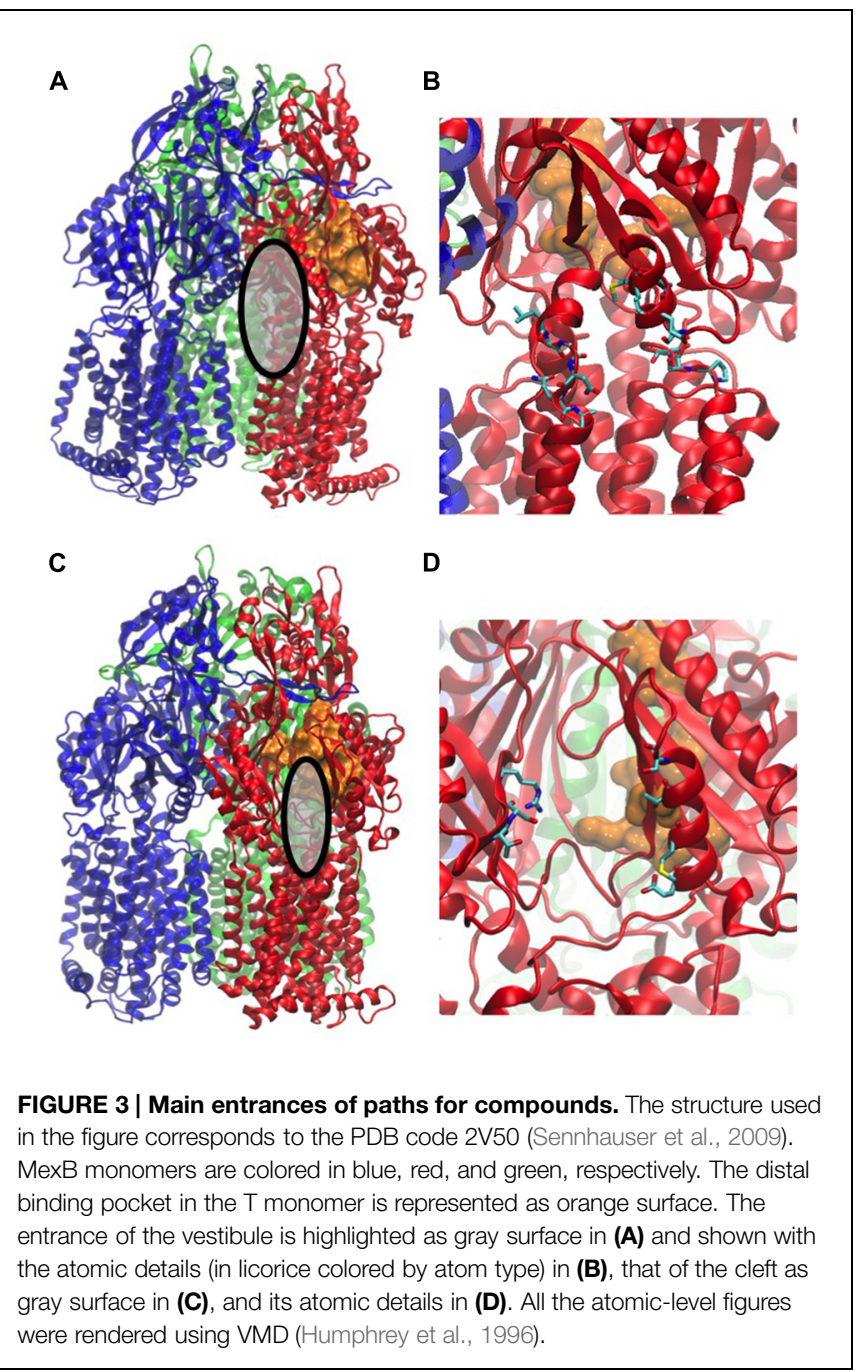

through the cleft path starting at a large external indentation of the periplasmic domain, formed by the subdomains PC1 and PC2 of a single AcrB protomer. The cleft path, whose entrance is shown in Figures 3C,D, directly connects the periplasm to the DP (Husain and Nikaido, 2010). Smaller drugs were found to favor the vestibule path, while larger compounds preferentially took the cleft path.

The direct simulations identified a novel alternative uptake path, which is not visible in the crystal structure. This third path goes along the bottom of the porter domain toward PC1 and could be validated by site-directed mutagenesis of AcrB in E. coli. Mutations of residues located along the path significantly impaired the efflux efficiency. The work of Yao et al. (2013) is of great interest because it combined different techniques to gain insight into an important step of the efflux process. However, it has to be noted that the authors made several approximations. As in all the computational studies described in this review, the partners of the RND transporter (i.e., AcrA and TolC in the present case) were missing. It was also assumed that the drug uptake was mainly driven by hydrophobic interactions. Finally, some of the physicochemical properties such as the ordering of amphiphilic drugs in the lipid bilayer and the conformational flexibility of drugs with rotatable bonds were neglected. Despite of the necessary approximations, the work of Yao et al. (2013) was an important contribution to the understanding of drug RND efflux. The structures of the L monomers and ligand conformations in co-crystals suggest interesting mechanistic differences between MexB and AcrB (Sennhauser et al., 2009; Nakashima et al., 2013). Thus, a similar study on MexB would be of great interest.

\section{Conclusion}

Much progress has been made in the understanding of the transport mechanism of RND efflux pumps since they were discovered. Genetic and biochemical investigations have provided a good survey of the substrate specificity of different pump complexes in various bacteria. It has become accepted that RND pumps evolved as a first line of bacterial defense against exogenous substances, allowing the development of additional defense strategies (Olivares et al., 2013). Molecules that are able to penetrate the $\mathrm{OM}$ are potentially problematic for Gram-negative bacteria. Many sophisticated studies have supported this view as they have indicated that amphiphilic molecules (such as many antibiotics), which are able to pass the $\mathrm{OM}$, are good substrates for export systems (Nikaido, 2011; Nikaido and Pages, 2012). $\mathrm{X}$-ray crystallography has provided highly detailed structural information on binding interactions between pump subunits and substrates (Ruggerone et al., 2013a). It is intriguing that only a handful of a large number of known efflux substrates could be cocrystallized with efflux pumps. Although X-ray structures provide snap-shots of a complex transport mechanism, they have been crucial for the setup of a transport model. Concerning specific experiments aiming at a more direct investigation of efflux dynamics, studies with whole bacteria have been performed. Whole cell efflux assays require thorough controls to rule out non-specific effects that interfere with transport measurements. Such control measurements include the use of membraneinteracting probes to monitor effects on membrane integrity or on the electrochemical potential across the inner membrane. The finding that many of the membrane-interacting probes are efflux substrates too, has confirmed the concept of recognition and export of membrane-active compounds. Based on experimental data, new in silico tools have been developed and brought to a point where they can reliably describe or even predict the dynamics of compound extrusion by RND pump subunits.

Substrate recognition by RND efflux pumps is a multifactorial process that can be measured by different methods as described in this article. MexB, for example, has a broad substrate spectrum including large (e.g., erythromycin) and charged (e.g., aztreonam, PA $\beta \mathrm{N}$ ) molecules (see Table 1). MexB transports hydrophobic dyes, many of which have been primarily used to study membrane properties, which indicates a preference of this pump for hydrophobic substrates. This is in agreement with the finding that the activity of the hydrophilic antibiotic ceftobiprole is not significantly affected by MexB. Crystal structures and MD simulations of MexB strongly suggested that the DP, lined with 
hydrophobic amino acid residues, is the main structural element for the recognition of hydrophobic elements (Collu et al., 2012b; Nakashima et al., 2013). However, hydrophobicity alone is not sufficient to explain substrate recognition by MexB. Imipenem and meropenem have a similar size and hydrophobicity (Table 1) but only meropenem is transported by MexB. A computational study revealed that imipenem, unlike meropenem, made no high affinity contacts to the AP or to the DP but instead made long-life interactions with solvent molecules inside MexB and eventually did not enter MexB (Collu et al., 2012b).

The challenge for future studies will be to combine the precision of X-ray structures with the functional relevance of whole cell studies. MD simulations based on experimental data provide a promising tool to complement, integrate and rationalize these data and to shed some light onto this problem.

Despite the advances outlined in the review, dissecting the molecular and conformational steps that regulate transport of substrates by RND pumps is still a very challenging and intriguing task and requires a very efficient interplay between techniques and approaches coming from different fields. The fate of a compound, governed by the action of an RND transporter (i.e., efficiently or poorly transported), is determined by the subtle balance of different molecular contributions that are not necessary large. The interaction of meropenem and imipenem with MexB described in the Section Computational Study is a good example: small differences in flexibility and hydrophobicity are suggested to make the former a good substrate and the latter a poor one. Computational methods can offer insight at a level of accuracy that is not reachable by a single experimental technique but they need a continuous feedback from experiments. Further crystallographic studies of RND pumps in complex with substrates and more accurate modeling techniques with extended simulation times for large proteins are needed in order to achieve full atomic pictures of the

\section{References}

Aires, J. R., and Nikaido, H. (2005). Aminoglycosides are captured from both periplasm and cytoplasm by the AcrD multidrug efflux transporter of Escherichia coli. J. Bacteriol. 187, 1923-1929. doi: 10.1128/JB.187.6.19231929.2005

Akama, H., Kanemaki, M., Yoshimura, M., Tsukihara, T., Kashiwagi, T., Yoneyama, H., et al. (2004a). Crystal structure of the drug discharge outer membrane protein, OprM, of Pseudomonas aeruginosa: dual modes of membrane anchoring and occluded cavity end. J. Biol. Chem. 279, 52816-52819. doi: 10.1074/jbc.C400445200

Akama, H., Matsuura, T., Kashiwagi, S., Yoneyama, H., Narita, S.-I., Tsukihara, T., et al. (2004b). Crystal structure of the membrane fusion protein, MexA, of the multidrug transporter in Pseudomonas aeruginosa. J. Biol. Chem. 279, 25939-25942. doi: 10.1074/jbc.C400164200

Amin, I. M., Richmond, G. E., Sen, P., Koh, T. H., Piddock, L. J., and Chua, K. L. (2013). A method for generating marker-less gene deletions in multidrugresistant Acinetobacter baumannii. BMC Microbiol. 13:158. doi: 10.1186/14712180-13-158

Angus, B. L., Carey, A. M., Caron, D. A., Kropinski, A. M., and Hancock, R. E. (1982). Outer membrane permeability in Pseudomonas aeruginosa: comparison of a wild-type with an antibiotic-supersusceptible mutant. Antimicrob. Agents Chemother. 21, 299-309. doi: 10.1128/AAC.21.2.299 entire tripartite complexes. Efflux kinetics might be affected by several factors that have to be included in the simulations but are not known a priori (Kinana et al., 2013). Computer simulations can integrate data from experiments on the molecular level and help to interpret data from whole cell assays (i.e., functional efflux pump ensembles).

The development of novel antibiotics that can bypass the effects of MDR pumps or the development of clinically useful EPI is still a challenging task. Understanding the mechanistic details and the structure-function relationship of bacterial efflux systems, as well as their regulation and the synergistic interactions between the pumps and other resistance mechanisms, is not only scientifically rewarding but can also stimulate applied research for effective new antibacterial drugs.

\section{Addendum in Proof}

While our manuscript was under review two comprehensive review articles by Li et al. (2015) and by Yamaguchi et al. (2015) were published that cover many aspects discussed in our article.

\section{Acknowledgments}

PR has received support from the Innovative Medicines Initiative Joint Undertaking under Grant Agreement $\mathrm{n}^{\circ} 115525$, resources, which are composed of financial contribution from the European Union's seventh framework program (FP7/20072013) and from EFPIA companies in kind contribution, as part of the Project TRANSLOCATION (http://www.imi.europa.eu/ content/translocation).

We want to thank Dr. Stefan Reinelt for help with the calculation of the data in Table 1 and Dr. Jonathan Thwaite for critically reading the document.

Baum, E. Z., Crespo-Carbone, S. M., Morrow, B. J., Davies, T. A., Foleno, B. D., He, W., et al. (2009). Effect of MexXY overexpression on ceftobiprole susceptibility in Pseudomonas aeruginosa. Antimicrob. Agents Chemother. 53, 2785-2790. doi: 10.1128/AAC.00018-09

Bavro, V. N., Pietras, Z., Furnham, N., Pérez-Cano, L., Fernández-Recio, J., Pei, X. Y., et al. (2008). Assembly and channel opening in a bacterial drug efflux machine. Mol. Cell. 30, 114-121. doi: 10.1016/j.molcel.2008.02.015

Biarnés, X., Bongarzone, S., Vargiu, A. V., Carloni, P., and Ruggerone, P. (2011). Molecular motions in drug design: the coming age of the metadynamics method. J. Comput. Aided Mol. Des. 25, 395-402. doi: 10.1007/s10822-0119415-3

Blair, J. M., Bavro, V. N., Ricci, V., Modi, N., Cacciotto, P., Kleinekathfer, U., et al. (2015a). AcrB drug-binding pocket substitution confers clinically relevant resistance and altered substrate specificity. Proc. Natl. Acad. Sci. U.S.A. 112, 3511-3516. doi: 10.1073/pnas.1419939112

Blair, J. M. A., Webber, M. A., Baylay, A. J., Ogbolu, D. O., and Piddock, L. J. V. (2015b). Molecular mechanisms of antibiotic resistance. Nat. Rev. Microbiol. 13, 42-51. doi: 10.1038/nrmicro3380

Blair, J. M., Richmond, G. E., and Piddock, L. J. (2014). Multidrug efflux pumps in Gram-negative bacteria and their role in antibiotic resistance. Fut. Microbiol. 9, 1165-1177. doi: 10.2217/fmb.14.66

Bohnert, J. A., Karamian, B., and Nikaido, H. (2010). Optimized Nile Red efflux assay of AcrAB-TolC multidrug efflux system shows competition 
between substrates. Antimicrob. Agents Chemother. 54, 3770-3775. doi: 10.1128/AAC.00620-10

Bohnert, J. A., Schuster, S., Seeger, M. A., Fähnrich, E., Pos, K. M., and Kern, W. V. (2008). Site-directed mutagenesis reveals putative substrate binding residues in the Escherichia coli RND efflux pump AcrB. J. Bacteriol. 190, 8225-8229. doi: 10.1128/JB.00912-08

Bohnert, J. A., Schuster, S., Szymaniak-Vits, M., and Kern, W. V. (2011a). Determination of real-time efflux phenotypes in Escherichia coli AcrB binding pocket phenylalanine mutants using a 1,2'-dinaphthylamine efflux assay. PLoS ONE 6:e21196. doi: 10.1371/journal.pone.0021196

Bohnert, J. A., Szymaniak-Vits, M., Schuster, S., and Kern, W. V. (2011b). Efflux inhibition by selective serotonin reuptake inhibitors in Escherichia coli. J. Antimicrob. Chemother. 66, 2057-2060. doi: 10.1093/jac/dkr258

Borges-Walmsley, M. I., Mckeegan, K. S., and Walmsley, A. R. (2003). Structure and function of efflux pumps that confer resistance to drugs. Biochem. J. 376, 313-338. doi: 10.1042/BJ20020957

Brown, D. G., May-Dracka, T. L., Gagnon, M. M., and Tommasi, R. (2014). Trends and exceptions of physical properties on antibacterial activity for Grampositive and Gram-negative pathogens. J. Med. Chem. 57, 10144-10161. doi: 10.1021/jm501552x

Butler, M. J., Bergeron, A., Soostmeyer, G., Zimny, T., and Malek, L. T. (1993). Cloning and characterisation of an aminopeptidase P-encoding gene from Streptomyces lividans. Gene 123, 115-119. doi: 10.1016/0378-1119(93)90549-I

Cai, H., Rose, K., Liang, L. H., Dunham, S., and Stover, C. (2009). Development of a liquid chromatography/mass spectrometry-based drug accumulation assay in Pseudomonas aeruginosa. Anal. Biochem. 385, 321-325. doi: 10.1016/j.ab.2008.10.041

Caiazza, N. C., Shanks, R. M., and O'toole, G. A. (2005). Rhamnolipids modulate swarming motility patterns of Pseudomonas aeruginosa. J. Bacteriol. 187, 73517361. doi: 10.1128/JB.187.21.7351-7361.2005

Castanheira, M., Deshpande, L. M., Costello, A., Davies, T. A., and Jones, R. N. (2014). Epidemiology and carbapenem resistance mechanisms of carbapenemnon-susceptible Pseudomonas aeruginosa collected during 2009-11 in 14 European and Mediterranean countries. J. Antimicrob. Chemother. 69, 18041814. doi: $10.1093 / \mathrm{jac} / \mathrm{dku} 048$

CDC.(2013). US Department Health and Human Services. Centers for Disease Control and Prevention. Antibiotic Resistance Threats in the United States, 2013. Available at: http://www.cdc.gov/drug resistance/pdf/arthreats-2013-508.pdf

Chen, J., Morita, Y., Huda, M. N., Kuroda, T., Mizushima, T., and Tsuchiya, T. (2002). VmrA a member of a novel class of $\mathrm{Na}^{+}$-coupled multidrug efflux pumps from Vibrio parahaemolyticus. J. Bacteriol. 184, 572-576. doi: $10.1128 / J B .184 .2 .572-576.2002$

Chipot, C. (2008). Free energy calculations applied to membrane proteins. Methods Mol. Biol. 443, 121-144. doi: 10.1007/978-1-59745-177-2_7

Chollet, R., Chevalier, J., Bryskier, A., and Pages, J. M. (2004). The AcrAB-TolC pump is involved in macrolide resistance but not in telithromycin efflux in Enterobacter aerogenes and Escherichia coli. Antimicrob. Agents Chemother. 48, 3621-3624. doi: 10.1128/AAC.48.9.3621-3624.2004

Chopra, I. (2002). New developments in tetracycline antibiotics: glycylcyclines and tetracycline efflux pump inhibitors. Drug Resist. Update 5, 119-125. doi: 10.1016/S1368-7646(02)00051-1

Chopra, I., and Roberts, M. (2001). Tetracycline antibiotics: mode of action, applications, molecular biology, and epidemiology of bacterial resistance. Microbiol. Mol. Biol. Rev. 65, 232-260. doi: 10.1128/MMBR.65.2.232260.2001

Collu, F., and Cascella, M. (2013). Multidrug resistance and efflux pumps: insights from molecular dynamics simulations. Curr. Top. Med. Chem. 13, 3165-3183. doi: $10.2174 / 15680266113136660224$

Collu, F., Ceccarelli, M., and Ruggerone, P. (2012a). Exploring binding properties of agonists interacting with a $\delta$-opioid receptor. PLOS ONE 7:e52633. doi: 10.1371/journal.pone.0052633

Collu, F., Vargiu, A. V., Dreier, J., Cascella, M., and Ruggerone, P. (2012b). Recognition of imipenem and meropenem by the Rnd-transporter MexB studied by computer simulations. J. Am. Chem. Soc. 134, 19146-19158. doi: 10.1021/ja307803m

Cowan, S. W., Schirmer, T., Rummel, G., Steiert, M., Ghosh, R., Pauptit, R. A., et al. (1992). Crystal structures explain functional properties of two E. coli porins. Nature 358, 727-733. doi: 10.1038/358727a0
Coyne, S., Rosenfeld, N., Lambert, T., Courvalin, P., and Perichon, B. (2010). Overexpression of resistance-nodulation-cell division pump AdeFGH confers multidrug resistance in Acinetobacter baumannii. Antimicrob. Agents Chemother. 54, 4389-4393. doi: 10.1128/AAC.00155-10

Dal Peraro, M., Ruggerone, P., Raugei, S., Gervasio, F. L., and Carloni, P. (2007). Investigating biological systems using first principles Car-Parrinello molecular dynamics simulations. Curr. Opin. Struc. Biol. 17, 149-156. doi: 10.1016/j.sbi.2007.03.018

Damier-Piolle, L., Magnet, S., Bremont, S., Lambert, T., and Courvalin, P. (2008). AdeIJK, a resistance-nodulation-cell division pump effluxing multiple antibiotics in Acinetobacter baumannii. Antimicrob. Agents Chemother. 52, 557-562. doi: 10.1128/AAC.00732-07

Davin-Regli, A., Bolla, J. M., James, C. E., Lavigne, J. P., Chevalier, J., Garnotel, E., et al. (2008). Membrane permeability and regulation of drug "influx and efflux" in enterobacterial pathogens. Curr. Drug Targets 9, 750-759. doi: 10.2174/138945008785747824

Dean, C. R., Visalli, M. A., Projan, S. J., Sum, P. E., and Bradford, P. A. (2003). Efflux-mediated resistance to tigecycline (GAR-936) in Pseudomonas aeruginosa PAO1. Antimicrob. Agents Chemother. 47, 972-978. doi: 10.1128/AAC.47.3.972-978.2003

Delcour, A. H. (2009). Outer membrane permeability and antibiotic resistance. Biochim. Biophys. Acta 1794, 808-816. doi: 10.1016/j.bbapap.2008. 11.005

Delmar, J. A., Su, C. C., and Yu, E. W. (2014). Bacterial multidrug efflux transporters. Annu. Rev. Biophys. 43, 93-117. doi: 10.1146/annurev-biophys051013-022855

Denys, G. A., and Relich, R. F. (2014). Antibiotic resistance in nosocomial respiratory infections. Clin. Lab. Med. 34, 257-270. doi: 10.1016/j.cll.2014.02.004

de Vries, S. J., and Zacharias, M. (2012). Attract-Em: a new method for the computational assembly of large molecular machines using cryo-EM maps. PLOS ONE 7:e49733. doi: 10.1371/journal.pone.0049733

Deziel, E., Lepine, F., Milot, S., and Villemur, R. (2003). rhlA is required for the production of a novel biosurfactant promoting swarming motility in Pseudomonas aeruginosa: 3-(3-hydroxyalkanoyloxy)alkanoic acids (HAAs), the precursors of rhamnolipids. Microbiology 149, 2005-2013. doi: 10.1099/mic.0.26154-0

Dreier, J. (2007). "Active Drug Efflux in Bacteria," in: Enzyme-Mediated Resistance to Antibiotics: Mechanisms, Dissemination, and Prospects for Inhibition, First Edn, ed. R. A. T. M. E. Bonomo (Washington, DC: ASM Press). doi: 10.1128/9781555815615.ch15

Du, D., Van Veen, H. W., and Luisi, B. F. (2015). Assembly and operation of bacterial tripartite multidrug efflux pumps. Trends Microbiol. 23, 311-319. doi: 10.1016/j.tim.2015.01.010

Du, D., Wang, Z., James, N. R., Voss, J. E., Klimont, E., Ohene-Agyei, T., et al. (2014). Structure of the AcrAB-TolC multidrug efflux pump. Nature 509, 512-515. doi: 10.1038/nature13205

Dunham, S. A., Mcpherson, C. J., and Miller, A. A. (2010). The relative contribution of efflux and target gene mutations to fluoroquinolone resistance in recent clinical isolates of Pseudomonas aeruginosa. Eur. J. Clin. Microbiol. Infect. Dis. 29, 279-288. doi: 10.1007/s10096-009-0852-z

ECDC. (2015). European Centre for Disease Prevention and Control. Annual Epidemiological Report 2014 Antimicrobial Resistance and HealthcareAssociated Infections, Stockholm.

Eda, S., Maseda, H., and Nakae, T. (2003). An elegant means of self-protection in gram-negative bacteria by recognizing and extruding xenobiotics from the periplasmic space. J. Biol. Chem. 278, 2085-2088. doi: 10.1074/jbc.C2006 61200

Eguchi, K., Ueda, Y., Kanazawa, K., Sunagawa, M., and Gotoh, N. (2007). The mode of action of 2-(thiazol-2-ylthio)-1beta-methylcarbapenems against Pseudomonas aeruginosa: the impact of outer membrane permeability and the contribution of MexAB-OprM efflux system. J. Antibiot. 60, 129-135. doi: 10.1038/ja.2007.12

Eicher, T., Brandstatter, L., and Pos, K. M. (2009). Structural and functional aspects of the multidrug efflux pump AcrB. Biol. Chem. 390, 693-699. doi: 10.1515/BC.2009.090

Eicher, T., Cha, H. J., Seeger, M. A., Brandstatter, L., El-Delik, J., Bohnert, J. A., et al. (2012). Transport of drugs by the multidrug transporter AcrB involves an access 
and a deep binding pocket that are separated by a switch-loop. Proc. Natl. Acad. Sci. U.S.A. 109, 5687-5692. doi: 10.1073/pnas.1114944109

Eicher, T., Seeger, M. A., Anselmi, C., Zhou, W., Brandstatter, L., Verrey, F., et al. (2014). Coupling of remote alternating-access transport mechanisms for protons and substrates in the multidrug efflux pump AcrB. Elife 19, 3. doi: 10.7554/eLife. 03145

Elkins, C. A., and Nikaido, H. (2002). Substrate specificity of the RND-type multidrug efflux pumps AcrB and AcrD of Escherichia coli is determined predominantly by two large periplasmic loops. J. Bacteriol. 184, 6490-6498. doi: 10.1128/JB.184.23.6490-6499.2002

Fernandez, L., and Hancock, R. E. (2012). Adaptive and mutational resistance: role of porins and efflux pumps in drug resistance. Clin. Microbiol. Rev. 25, 661-681. doi: 10.1128/CMR.00043-12

Ferrandez, Y., Monlezun, L., Phan, G., Benabdelhak, H., Benas, P., Ulryck, N., et al. (2012). Stoichiometry of the MexA-OprM binding, as investigated by blue native gel electrophoresis. Electrophoresis 33, 1282-1287. doi: 10.1002/elps.201100541

Ferreira, M. M. C., and Kiralj, R. (2004). QSAR study of B-lactam antibiotic efflux by the multidrug resistance pump AcrB. J. Chemometr. 18, 242-252. doi: $10.1002 / \mathrm{cem} .867$

Fieldler, F., and Hinz, H. (1994). No intermediate channelling in stepwise hydrolysis of fluorescein di-beta-D-galactoside by beta-galactosidase. Eur. J. Biochem. 222, 75-81. doi: 10.1111/j.1432-1033.1994.tb18843.x

Fischer, N., and Kandt, C. (2011). Three ways in, one way out: water dynamics in the trans-membrane domains of the inner membrane translocase AcrB. Proteins 79, 2871-2885. doi: 10.1002/prot.23122

Fischer, N., and Kandt, C. (2012). Porter domain opening and closing motions in the multi-drug efflux transporter AcrB. Biochim. Biophys. Acta 1828, 632-641. doi: 10.1016/j.bbamem.2012.10.016

Fischer, N., Raunest, M., Schmidt, T. H., Koch, D. C., and Kandt, C. (2014). Efflux pump-mediated antibiotics resistance: insights from computational structural biology. Interdiscip. Sci. 6, 1-12. doi: 10.1007/s12539-0140191-3

Fukuda, H., Hosaka, M., Hirai, K., and Iyobe, S. (1990). New norfloxacin resistance gene in Pseudomonas aeruginosa Pao. Antimicrob. Agents Chemother. 34, 17571761. doi: 10.1128/AAC.34.9.1757

Fukuda, H., Hosaka, M., Iyobe, S., Gotoh, N., Nishino, T., and Hirai, K. (1995). nfxC-type quinolone resistance in a clinical isolate of Pseudomonas aeruginosa. Antimicrob. Agents Chemother. 39, 790-792. doi: 10.1128/AAC.39. 3.790

Garvey, M. I., and Piddock, L. J. (2008). The efflux pump inhibitor reserpine selects multidrug-resistant Streptococcus pneumoniae strains that overexpress the ABC transporters PatA and PatB. Antimicrob. Agents Chemother. 52, 1677-1685. doi: 10.1128/AAC.01644-07

Germ, M., Yoshihara, E., Yoneyama, H., and Nakae, T. (1999). Interplay between the efflux pump and the outer membrane permeability barrier in fluorescent dye accumulation in Pseudomonas aeruginosa. Biochem. Biophys. Res. Commun. 261, 452-455. doi: 10.1006/bbrc.1999.1045

Giske, C. G., Boren, C., Wretlind, B., and Kronvall, G. (2005). Meropenem susceptibility breakpoint for Pseudomonas aeruginosa strains hyperproducing mexB mRNA. Clin. Microbiol. Infect. 11, 662-669. doi: 10.1111/j.14690691.2005.01182x

Gonzalez, R. J., and Tarloff, J. B. (2001). Evaluation of hepatic subcellular fractions for Alamar blue and MTT reductase activity. Toxicol. In Vitro 15, 257-259. doi: 10.1016/S0887-2333(01)00014-5

Greenspan, P., and Fowler, S. D. (1985). Spectrofluorometric studies of the lipid probe, nile red. J. Lipid Res. 26, 781-789.

Griffith, D. C., Corcoran, E., Lofland, D., Lee, A., Cho, D., Lomovskaya, O., et al. (2006). Pharmacodynamics of levofloxacin against Pseudomonas aeruginosa with reduced susceptibility due to different efflux pumps: do elevated MICs always predict reduced in vivo efficacy? Antimicrob. Agents Chemother. 50, 1628-1632. doi: 10.1128/AAC.50.5.1628-1632.2006

Grkovic, S., Brown, M. H., and Skurray, R. A. (2002). Regulation of bacterial drug export systems. Microbiol. Mol. Biol. Rev. 66, 671-701. doi: 10.1128/MMBR.66.4.671-701.2002

Hancock, R. E. (1997). The bacterial outer membrane as a drug barrier. Trends Microbiol. 5, 37-42. doi: 10.1016/S0966-842X(97)81773-8
Hayashi, K., Fukushima, A., Hayashi-Nishino, M., and Nishino, K. (2014) Effect of methylglyoxal on multidrug-resistant Pseudomonas aeruginosa. Front. Microbiol. 5:180. doi: 10.3389/fmicb.2014.00180

Hidron, A. I., Edwards, J. R., Patel, J., Horan, T. C., Sievert, D. M., Pollock, D. A., et al. (2008). NHSN annual update: antimicrobial-resistant pathogens associated with healthcare-associated infections: annual summary of data reported to the National Healthcare Safety Network at the Centers for Disease Control and Prevention, 2006-2007. Infect. Control. Hosp. Epidemiol. 29, 9961011. doi: $10.1086 / 591861$

Hinchliffe, P., Symmons, M. F., Hughes, C., and Koronakis, V. (2013). Structure and operation of bacterial tripartite pumps. Annu. Rev. Microbiol. 67, 221-242. doi: 10.1146/annurev-micro-092412-155718

Hirata, T., Saito, A., Nishino, K., Tamura, N., and Yamaguchi, A. (2004). Effects of efflux transporter genes on susceptibility of Escherichia coli to tigecycline (GAR-936). Antimicrob. Agents Chemother. 48, 2179-2184. doi: 10.1128/AAC.48.6.2179-2184.2004

Hocquet, D., Nordmann, P., El Garch, F., Cabanne, L., and Plesiat, P. (2006). Involvement of the MexXY-OprM efflux system in emergence of cefepime resistance in clinical strains of Pseudomonas aeruginosa. Antimicrob. Agents Chemother. 50, 1347-1351. doi: 10.1128/AAC.50.4.1347-1351.2006

Humphrey, W., Dalke, A., and Schulten, K. (1996). Vmd: visual molecular dynamics. J. Mol. Graph. 14, 33-38. doi: 10.1016/0263-7855(96)00018-5

Husain, F., and Nikaido, H. (2010). Substrate path in the AcrB multidrug efflux pump of Escherichia coli. Mol. Microbiol. 78, 320-330. doi: 10.1111/j.13652958.2010.07330.x

Iino, R., Hayama, K., Amezawa, H., Sakakihara, S., Kim, S. H., Matsumono, Y., et al. (2012a). A single-cell drug efflux assay in bacteria by using a directly accessible femtoliter droplet array. Lab. Chip. 12, 3923-3929. doi: 10.1039/c2lc40394c

Iino, R., Nishino, K., Noji, H., Yamaguchi, A., and Matsumoto, Y. (2012b). A microfluidic device for simple and rapid evaluation of multidrug efflux pump inhibitors. Front. Microbiol. 3:40. doi: 10.3389/fmicb.2012.00040

Iino, R., Matsumoto, Y., Nishino, K., Yamaguchi, A., and Noji, H. (2013). Design of a large-scale femtoliter droplet array for single-cell analysis of drug-tolerant and drug-resistant bacteria. Front. Microbiol. 4:300. doi: 10.3389/fmicb.2013.00300

Jones, R. N., Stilwell, M. G., Rhomberg, P. R., and Sader, H. S. (2009). Antipseudomonal activity of piperacillin/tazobactam: more than a decade of experience from the SENTRY Antimicrobial Surveillance Program (19972007). Diagn. Microbiol. Infect. Dis. 65, 331-334. doi: 10.1016/j.diagmicrobio. 2009.06.022

Kamal, M. Z., Ali, J., and Rao, N. M. (2013). Binding of bis-ANS to Bacillus subtilis lipase: a combined computational and experimental investigation. Biochim. Biophys. Acta 1834, 1501-1509. doi: 10.1016/j.bbapap.2013.04.021

Kamerlin, S. C., Vicatos, S., Dryga, A., and Warshel, A. (2011). Coarse-grained (multiscale) simulations in studies of biophysical and chemical systems. Annu. Rev. Phys. Chem. 62, 41-64. doi: 10.1146/annurev-physchem-032210103335

Kamio, Y., and Nikaido, H. (1976). Outer membrane of Salmonella typhimurium: accessibility of phospholipid head groups to phospholipase $c$ and cyanogen bromide activated dextran in the external medium. Biochemistry 15, 2561-2570. doi: $10.1021 / \mathrm{bi00657a012}$

Karplus, M. (2014). Development of multiscale models for complex chemical systems: from $\mathrm{H}^{+} \mathrm{H}(2)$ to biomolecules (Nobel Lecture). Angew. Chem. Int. Ed. Engl. 53, 9992-10005. doi: 10.1002/anie.201403924

Kim, J. S., Jeong, H., Song, S., Kim, H.-Y., Lee, K., Hyun, J., et al. (2015). Structure of the tripartite multidrug efflux pump acrab-TolC suggests an alternative assembly mode. Mol. Cells 38, 180-186. doi: 10.14348/molcells.2015.2277

Kinana, A. D., Vargiu, A. V., and Nikaido, H. (2013). Some ligands enhance the efflux of other ligands by the Escherichia coli multidrug pump AcrB. Biochemistry 52, 8342-8351. doi: 10.1021/bi401303v

Kobayashi, N., Tamura, N., Van Veen, H. W., Yamaguchi, A., and Murakami, S. (2014). beta-Lactam selectivity of multidrug transporters AcrB and AcrD resides in the proximal binding pocket. J. Biol. Chem. 289, 10680-10690. doi: 10.1074/jbc.M114.547794

Kohler, T., Kok, M., Michea-Hamzehpour, M., Plesiat, P., Gotoh, N., Nishino, T., et al. (1996). Multidrug efflux in intrinsic resistance to trimethoprim and sulfamethoxazole in Pseudomonas aeruginosa. Antimicrob. Agents Chemother. $40,2288-2290$ 
Kohler, T., Michea-Hamzehpour, M., Plesiat, P., Kahr, A. L., and Pechere, J. C. (1997). Differential selection of multidrug efflux systems by quinolones in Pseudomonas aeruginosa. Antimicrob. Agents Chemother. 41, 2540-2543.

Kollman, P. A., Massova, I., Reyes, C., Kuhn, B., Huo, S., Chong, L., et al. (2000). Calculating structures and free energies of complex molecules: combining molecular mechanics and continuum models. Acc. Chem. Res. 33, 889-897. doi: 10.1021/ar000033j

Koronakis, V., Eswaran, J., and Hughes, C. (2004). Structure and function of TolC: the bacterial exit duct for proteins and drugs. Annu. Rev. Biochem. 73, 467-489. doi: 10.1146/annurev.biochem.73.011303.074104

Koronakis, V., Sharff, A., Koronakis, E., Luisi, B., and Hughes, C. (2000). Crystal structure of the bacterial membrane protein TolC central to multidrug efflux and protein export. Nature 405, 914-919. doi: 10.1038/35016007

Kriengkauykiat, J., Porter, E., Lomovskaya, O., and Wong-Beringer, A. (2005). Use of an efflux pump inhibitor to determine the prevalence of efflux pump-mediated fluoroquinolone resistance and multidrug resistance in Pseudomonas aeruginosa. Antimicrob. Agents Chemother. 49, 565-570. doi: 10.1128/AAC.49.2.565-570.2005

Krishnamoorthy, G., Tikhonova, E. B., Dhamdhere, G., and Zgurskaya, H. I. (2013). On the role of TolC in multidrug efflux: the function and assembly of AcrAB-TolC tolerate significant depletion of intracellular TolC protein. Mol. Microbiol. 87, 982-997. doi: 10.1111/mmi.12143

Krishnamoorthy, G., Tikhonova, E. B., and Zgurskaya, H. I. (2008). Fitting periplasmic membrane fusion proteins to inner membrane transporters: mutations that enable Escherichia coli AcrA to function with Pseudomonas aeruginosa MexB. J. Bacteriol. 190, 691-698. doi: 10.1128/JB.01276-07

Kumar, A., Chua, K. L., and Schweizer, H. P. (2006). Method for regulated expression of single-copy efflux pump genes in a surrogate Pseudomonas aeruginosa strain: identification of the BpeEF-OprC chloramphenicol and trimethoprim efflux pump of Burkholderia pseudomallei 1026b. Antimicrob. Agents Chemother. 50, 3460-3463. doi: 10.1128/AAC.00440-06

Kumar, A., and Schweizer, H. P. (2005). Bacterial resistance to antibiotics: active efflux and reduced uptake. Adv. Drug Deliv. Rev. 57, 1486-1513. doi: 10.1016/j.addr.2005.04.004

Laio, A., and Gervasio, F. L. (2008). Metadynamics: a method to simulate rare events and reconstruct the free energy in biophysics, chemistry and material science. Rep. Prog. Phys. 71, 126601. doi: 10.1088/0034-4885/71/12/ 126601

Laio, A., Rodriguez-Fortea, A., Gervasio, F. L., Ceccarelli, M., and Parrinello, M. (2005). Assessing the accuracy of metadynamics. J. Phys. Chem. B 109, 67146721. doi: $10.1021 / j p 045424 \mathrm{k}$

Lamers, R. P., Cavallari, J. F., and Burrows, L. L. (2013). The efflux inhibitor phenylalanine-arginine beta-naphthylamide (PabetaN) permeabilizes the outer membrane of gram-negative bacteria. PLoS ONE 8:e60666. doi: 10.1371/journal.pone.0060666

Lau, C. H., Hughes, D., and Poole, K. (2014). MexY-promoted aminoglycoside resistance in Pseudomonas aeruginosa: involvment of a putative proximal binding pocket in aminoglycoside recognition. MBio 5:e01068. doi: 10.1128/mBio.01068-4

LePecq, J. B., and Paoletti, C. (1967). A fluorescent complex between ethidium bromide and nucleic acids. Physical-chemical characterization. J. Mol. Biol. 27, 87-106. doi: 10.1016/0022-2836(67)90353-1

Levy, S. B. (1992). Active efflux mechanisms for antimicrobial resistance. Antimicrob. Agents Chemother. 36, 695-703. doi: 10.1128/AAC.36.4.695

Li, X. Z., Ma, D., Livermore, D. M., and Nikaido, H. (1994). Role of efflux pump(s) in intrinsic resistance of Pseudomonas aeruginosa: active efflux as a contributing factor to beta-lactam resistance. Antimicrob. Agents Chemother. 38, 1742-1752. doi: 10.1128/AAC.38.8.1742

Li, X. Z., Nikaido, H., and Poole, K. (1995). Role of mexA-mexB-oprM in antibiotic efflux in Pseudomonas aeruginosa. Antimicrob. Agents Chemother. 39, 19481953. doi: 10.1128/AAC.39.9.1948

Li, X. Z., Plesiat, P., and Nikaido, H. (2015). The challenge of efflux-mediated antibiotic resistance in Gram-negative bacteria. Clin. Microbiol. Rev. 28, 337418. doi: 10.1128/CMR.00117-14

Li, Y., Mima, T., Komori, Y., Morita, Y., Kuroda, T., Mizushima, T., et al. (2003). A new member of the tripartite multidrug efflux pumps, MexVWOprM, in Pseudomonas aeruginosa. J. Antimicrob. Chemother. 52, 572-575. doi: $10.1093 / \mathrm{jac} / \mathrm{dkg} 390$
Lister, P. D., Wolter, D. J., and Hanson, N. D. (2009). Antibacterialresistant Pseudomonas aeruginosa: clinical impact and complex regulation of chromosomally encoded resistance mechanisms. Clin. Microbiol. Rev. 22, 582-610. doi: 10.1128/CMR.00040-09

Livermore, D. M. (2009). Has the era of untreatable infections arrived? J. Antimicrob. Chemother. 64(Suppl. 1), i29-i36. doi: 10.1093/jac/dkp255

Loh, B., Grant, C., and Hancock, R. E. (1984). Use of the fluorescent probe 1-Nphenylnaphthylamine to study the interactions of aminoglycoside antibiotics with the outer membrane of Pseudomonas aeruginosa. Antimicrob. Agents Chemother. 26, 546-551. doi: 10.1128/AAC.26.4.546

Lomovskaya, O., and Bostian, K. A. (2006). Practical applications and feasibility of efflux pump inhibitors in the clinic-a vision for applied use. Biochem. Pharmacol. 71, 910-918. doi: 10.1016/j.bcp.2005.12.008

Lomovskaya, O., Lee, A., Hoshino, K., Ishida, H., Mistry, A., Warren, M. S., et al. (1999). Use of a genetic approach to evaluate the consequences of inhibition of efflux pumps in Pseudomonas aeruginosa. Antimicrob. Agents Chemother. 43, 1340-1346.

Lomovskaya, O., Warren, M. S., Lee, A., Galazzo, J., Fronko, R., Lee, M., et al. (2001). Identification and characterization of inhibitors of multidrug resistance efflux pumps in Pseudomonas aeruginosa: novel agents for combination therapy. Antimicrob. Agents Chemother. 45, 105-116. doi: 10.1128/AAC.45.1. 105-116.2001

Loontiens, F. G., Regenfuss, P., Zechel, A., Dumortier, L., and Clegg, R. M. (1990). Binding characteristics of Hoechst 33258 with calf thymus DNA, poly[d(AT)], and d(CCGGAATTCCGG): multiple stoichiometries and determination of tight binding with a wide spectrum of site affinities. Biochemistry 29, 9029-9039. doi: 10.1021/bi00490a021

Ma, D., Cook, D. N., Alberti, M., Pon, N. G., Nikaido, H., and Hearst, J. E. (1993). Molecular cloning and characterization of acrA and acrE genes of Escherichia coli. J. Bacteriol. 175, 6299-6313.

Magnet, S., Courvalin, P., and Lambert, T. (2001). Resistance-nodulationcell division-type efflux pump involved in aminoglycoside resistance in Acinetobacter baumannii strain BM4454. Antimicrob. Agents Chemother. 45, 3375-3380. doi: 10.1128/AAC.45.12.3375-3380.2001

Mahamoud, A., Chevalier, J., Alibert-Franco, S., Kern, W. V., and Pages, J. M. (2007). Antibiotic efflux pumps in Gram-negative bacteria: the inhibitor response strategy. J. Antimicrob. Chemother. 59, 1223-1229. doi: $10.1093 / \mathrm{jac} / \mathrm{dkl} 493$

Mao, W., Warren, M. S., Black, D. S., Satou, T., Murata, T., Nishino, T., et al. (2002). On the mechanism of substrate specificity by resistance nodulation division (RND)-type multidrug resistance pumps: the large periplasmic loops of MexD from Pseudomonas aeruginosa are involved in substrate recognition. Mol. Microbiol. 46, 889-901. doi: 10.1046/j.1365-2958.2002. 03223.x

Mao, W., Warren, M. S., Lee, A., Mistry, A., and Lomovskaya, O. (2001). MexXYOprM efflux pump is required for antagonism of aminoglycosides by divalent cations in Pseudomonas aeruginosa. Antimicrob. Agents Chemother. 45, 20012007. doi: 10.1128/AAC.45.7.2001-2007.2001

Marks, N., Suhar, A., and Benuck, M. (1981). Peptide processing in the central nervous system. Adv. Biochem. Psychopharmacol. 28, 49-60.

Markwick, P. R., and McCammon, J. A. (2011). Studying functional dynamics in bio-molecules using accelerated molecular dynamics. Phys. Chem. Chem. Phys. 13, 20053-20065. doi: 10.1039/clcp22100k

Martinez, J. L., and Baquero, F. (2014). Emergence and spread of antibiotic resistance: setting a parameter space. Ups. J. Med. Sci. 119, 68-77. doi: 10.3109/ 03009734.2014.901444

Maseda, H., Yoneyama, H., and Nakae, T. (2000). Assignment of the substrate-selective subunits of the MexEF-OprN multidrug efflux pump of Pseudomonas aeruginosa. Antimicrob. Agents Chemother. 44, 658-664. doi: 10.1128/AAC.44.3.658-664.2000

Mason, E. O. Jr., Wald, E. R., Bradley, J. S., Barson, W. J., and Kaplan, S. L. (2003). Macrolide resistance among middle ear isolates of Streptococcus pneumoniae observed at eight United States pediatric centers: prevalence of $\mathrm{M}$ and MLSB phenotypes. Pediatr. Infect. Dis. J. 22, 623-627. doi: 10.1097/01.inf.0000073124.06415.93

Masuda, N., Gotoh, N., Ohya, S., and Nishino, T. (1996). Quantitative correlation between susceptibility and OprJ production in NfxB mutants of Pseudomonas aeruginosa. Antimicrob. Agents Chemother. 40, 909-913. 
Masuda, N., and Ohya, S. (1992). Cross-resistance to meropenem, cephems, and quinolones in Pseudomonas aeruginosa. Antimicrob. Agents Chemother. 36, 1847-1851. doi: 10.1128/AAC.36.9.1847

Masuda, N., Sakagawa, E., Ohya, S., Gotoh, N., and Nishino, T. (2001). Hypersusceptibility of the Pseudomonas aeruginosa $\mathrm{nfxB}$ mutant to betalactams due to reduced expression of the ampC beta-lactamase. Antimicrob. Agents Chemother. 45, 1284-1286. doi: 10.1128/AAC.45.4.1284-1286. 2001

Masuda, N., Sakagawa, E., Ohya, S., Gotoh, N., Tsujimoto, H., and Nishino, T. (2000a). Contribution of the MexX-MexY-oprM efflux system to intrinsic resistance in Pseudomonas aeruginosa. Antimicrob. Agents Chemother. 44, 2242-2246. doi: 10.1128/AAC.44.9.2242-2246.2000

Masuda, N., Sakagawa, E., Ohya, S., Gotoh, N., Tsujimoto, H., and Nishino, T. (2000b). Substrate specificities of MexAB-OprM, MexCD-OprJ, and MexXYoprM efflux pumps in Pseudomonas aeruginosa. Antimicrob. Agents Chemother. 44, 3322-3327. doi: 10.1128/AAC.44.12.3322-3327.2000

Matsumoto, Y., Hayama, K., Sakakihara, S., Nishino, K., Noji, H., Iino, R., et al. (2011). Evaluation of multidrug efflux pump inhibitors by a new method using microfluidic channels. PLOS ONE 6:e18547. doi: 10.1371/journal.pone.0018547

May, A., Sieker, F., and Zacharias, M. (2008). How to efficiently include receptor flexibility during computational docking. Curr. Comput. Aided Drug Design 4 143-153. doi: 10.2174/157340908784533265

May, A., and Zacharias, M. (2008). Protein-ligand docking accounting for receptor side chain and global flexibility in normal modes: evaluation on kinase inhibitor cross docking. J. Med. Chem. 51, 3499-3506. doi: 10.1021/jm800071v

Mazzariol, A., Cornaglia, G., and Nikaido, H. (2000). Contributions of the AmpC beta-lactamase and the AcrAB multidrug efflux system in intrinsic resistance of Escherichia coli K-12 to beta-lactams. Antimicrob. Agents Chemother. 44, 1387-1390. doi: 10.1128/AAC.44.5.1387-1390.2000

Mesaros, N., Glupczynski, Y., Avrain, L., Caceres, N. E., Tulkens, P. M., and Van Bambeke, F. (2007). A combined phenotypic and genotypic method for the detection of Mex efflux pumps in Pseudomonas aeruginosa. J. Antimicrob Chemother. 59, 378-386. doi: 10.1093/jac/dkl504

Middlemiss, J. K., and Poole, K. (2004). Differential impact of MexB mutations on substrate selectivity of the MexAB-OprM multidrug efflux pump of Pseudomonas aeruginosa. J. Bacteriol. 186, 1258-1269. doi: 10.1128/JB.186.5.1258-1269.2004

Mikolosko, J., Bobyk, K., Zgurskaya, H. I., and Ghosh, P. (2006). Conformational flexibility in the multidrug efflux system protein AcrA. Structure 14, 577-587. doi: 10.1016/j.str.2005.11.015

Mine, T., Morita, Y., Kataoka, A., Mizushima, T., and Tsuchiya, T. (1999). Expression in Escherichia coli of a new multidrug efflux pump, MexXY, from Pseudomonas aeruginosa. Antimicrob. Agents Chemother. 43, 415-417.

Misra, R., and Bavro, V. N. (2009). Assembly and transport mechanism of tripartite drug efflux systems. Bba Proteins Proteom 1794, 817-825. doi: 10.1016/j.bbapap.2009.02.017

Misra, R, Morrison, K. D., Cho, H. J., and Khuu, T. (2015). Imortance of real-time assays to distinguish multidrug efflux pump inhibiting and outer membrane destabilizing activities in Escherichia coli. J. Bacteriol. doi: 10.1128/JB.02456-4 [Epub ahead of print].

Morita, Y., Komori, Y., Mima, T., Kuroda, T., Mizushima, T., and Tsuchiya, T. (2001). Construction of a series of mutants lacking all of the four major mex operons for multidrug efflux pumps or possessing each one of the operons from Pseudomonas aeruginosa PAO1: MexCD-OprJ is an inducible pump. FEMS Microbiol. Lett. 202, 139-143. doi: 10.1111/j.1574-6968.2001.tb10794.x

Morita, Y., Murata, T., Mima, T., Shiota, S., Kuroda, T., Mizushima, T., et al. (2003). Induction of mexcd-oprJ operon for a multidrug efflux pump by disinfectants in wild-type Pseudomonas aeruginosa PAO1. J. Antimicrob. Chemother. 51, 991-994. doi: 10.1093/jac/dkg173

Morita, Y., Tomida, J., and Kawamura, Y. (2012). MexXY multidrug efflux system of Pseudomonas aeruginosa. Front. Microbiol. 3:408. doi: 10.3389/fmicb.2012.00408

Morita, Y., Tomida, J., and Kawamura, Y. (2014). Responses of Pseudomonas aeruginosa to antimicrobials. Front. Microbiol. 4:422. doi: $10.3389 /$ fmicb.2013.00422

Morita, Y., Tomida, J., and Kawamura, Y. (2015). Efflux-mediated fluoroquinolone resistance in the multidrug-resistant Pseudomonas aeruginosa clinical isolate PA7: identification of a novel MexS variant involved in upregulation of the mexef-oprN multidrug efflux operon. Front. Microbiol. 6:8. doi: 10.3389/fmicb.2015.00008

Morris, J. D., Hewitt, J. L., Wolfe, L. G., Kamatkar, N. G., Chapman, S. M., Diener, J. M., et al. (2011). Imaging and analysis of Pseudomonas aeruginosa swarming and rhamnolipid production. Appl. Environ. Microbiol. 77, 8310-8317. doi: 10.1128/AEM.06644-11

Mortimer, P. G., and Piddock, L. J. (1991). A comparison of methods used for measuring the accumulation of quinolones by Enterobacteriaceae, Pseudomonas aeruginosa and Staphylococcus aureus. J. Antimicrob. Chemother. 28, 639-653. doi: $10.1093 / \mathrm{jac} / 28.5 .639$

Murakami, S. (2008). Multidrug efflux transporter, AcrB-the pumping mechanism. Curr. Opin. Struct. Biol. 18, 459-465. doi: 10.1016/j.sbi.2008.06.007

Murakami, S., Nakashima, R., Yamashita, E., Matsumoto, T., and Yamaguchi, A. (2006). Crystal structures of a multidrug transporter reveal a functionally rotating mechanism. Nature 443, 173-179. doi: 10.1038/nature05076

Murakami, S., Nakashima, R., Yamashita, E., and Yamaguchi, A. (2002). Crystal structure of bacterial multidrug efflux transporter AcrB. Nature 419, 587-593. doi: $10.1038 /$ nature 01050

Murakami, S., Tamura, N., Saito, A., Hirata, T., and Yamaguchi, A. (2004). Extramembrane central pore of multidrug exporter AcrB in Escherichia coli plays an important role in drug transport. J. Biol. Chem. 279, 3743-3748. doi: 10.1074/jbc.M308893200

Nagano, K., and Nikaido, H. (2009). Kinetic behavior of the major multidrug efflux pump AcrB of Escherichia coli. Proc. Natl. Acad. Sci. U.S.A. 106, 5854-5858. doi: 10.1073/pnas.0901695106

Nakashima, R., Sakurai, K., Yamasaki, S., Hayashi, K., Nagata, C., Hoshino, K., et al. (2013). Structural basis for the inhibition of bacterial multidrug exporters. Nature 500, 102-106. doi: 10.1038/nature12300

Nakashima, R., Sakurai, K., Yamasaki, S., Nishino, K., and Yamaguchi, A. (2011). Structures of the multidrug exporter AcrB reveal a proximal multisite drugbinding pocket. Nature 480, 565-569. doi: 10.1038/nature10641

Nakayama, K., Ishida, Y., Ohtsuka, M., Kawato, H., Yoshida, K., Yokomizo, Y., et al. (2003). MexAB-OprM-specific efflux pump inhibitors in Pseudomonas aeruginosa. Part 1: discovery and early strategies for lead optimization. Bioorg. Med. Chem. Lett. 13, 4201-4204. doi: 10.1016/j.bmcl.2003.07.024

Narita, S., Eda, S., Yoshihara, E., and Nakae, T. (2003). Linkage of the efflux-pump expression level with substrate extrusion rate in the MexAB-OprM efflux pump of Pseudomonas aeruginosa. Biochem. Biophys. Res. Commun. 308, 922-926. doi: 10.1016/S0006-291X(03)01512-2

Nikaido, H. (1996). Multidrug efflux pumps of gram-negative bacteria. J. Bacteriol. $178,5853-5859$.

Nikaido, H. (2003). Molecular basis of bacterial outer membrane permeability revisited. Microbiol. Mol. Biol. Rev. 67, 593-656. doi: 10.1128/MMBR.67.4.593656.2003

Nikaido, H. (2011). Structure and mechanism of RND-type multidrug efflux pumps. Adv. Enzymol. Relat. Areas Mol. Biol. 77, 1-60. doi: 10.1002/9780470 920541.ch1

Nikaido, H., Basina, M., Nguyen, V., and Rosenberg, E. Y. (1998). Multidrug efflux pump AcrAB of Salmonella typhimurium excretes only those beta-lactam antibiotics containing lipophilic side chains. J. Bacteriol. 180, 4686-4692.

Nikaido, H., and Normark, S. (1987). Sensitivity of Escherichia coli to various beta-lactams is determined by the interplay of outer membrane permeability and degradation by periplasmic beta-lactamases: a quantitative predictive treatment. Mol. Microbiol. 1, 29-36. doi: 10.1111/j.1365-2958.1987. tb00523.x

Nikaido, H., and Pages, J. M. (2012). Broad-specificity efflux pumps and their role in multidrug resistance of Gram-negative bacteria. FEMS Microbiol. Rev. 36, 340-363. doi: 10.1111/j.1574-6976.2011.00290.x

Nikaido, H., and Rosenberg, E. Y. (1983). Porin channels in Escherichia coli: studies with liposomes reconstituted from purified proteins. J. Bacteriol. 153, 241-252.

Nishino, K., Nikaido, E., and Yamaguchi, A. (2009). Regulation and physiological function of multidrug efflux pumps in Escherichia coli and Salmonella. Biochim. Biophys. Acta 1794, 834-843. doi: 10.1016/j.bbapap.2009.02.002

Nishino, K., and Yamaguchi, A. (2002). EvgA of the two-component signal transduction system modulates production of the yhiuv multidrug transporter in Escherichia coli. J. Bacteriol. 184, 2319-2323. doi: 10.1128/JB.184.8.23192323.2002 
Ntsogo-Enguene, V. Y., Verchère, A., Phan, G., Broutin, I., and Picard, M. (2015). Catch me if you can: a biotinylated proteoliposome affinity assay for the investigation of assembly of the MexA-MexB-OprM efflux pump from Pseudomonas aeruginosa. Front. Microbiol. 6:541. doi: 10.3389/fmicb. 2015.00541

Ocaktan, A., Yoneyama, H., and Nakae, T. (1997). Use of fluorescence probes to monitor function of the subunit proteins of the MexA-MexB-oprM drug extrusion machinery in Pseudomonas aeruginosa. J. Biol. Chem. 272, 2196421969. doi: 10.1074/jbc.272.35.21964

Ohene-Agyei, T., Lea, J. D., and Venter, H. (2012). Mutations in MexB that affect the efflux of antibiotics with cytoplasmic targets. FEMS Microbiol. Lett. 333, 20-27. doi: 10.1111/j.1574-6968.2012.02594.x

Olaitan, A. O., Morand, S., and Rolain, J. M. (2014). Mechanisms of polymyxin resistance: acquired and intrinsic resistance in bacteria. Front. Microbiol. 5:643. doi: $10.3389 /$ fmicb.2014.00643

Olivares, J., Bernardini, A., Garcia-Leon, G., Corona, F. M. B. S., and Martinez, J. L. (2013). The intrinsic resistome of bacterial pathogens. Front. Microbiol. 4:103. doi: $10.3389 /$ fmicb. 2013.00103

Ong, C. T., Tessier, P. R., Li, C., Nightingale, C. H., and Nicolau, D. P. (2007). Comparative in vivo efficacy of meropenem, imipenem, and cefepime against Pseudomonas aeruginosa expressing MexA-MexB-OprM efflux pumps. Diagn. Microbiol. Infect. Dis. 57, 153-161. doi: 10.1016/j.diagmicrobio.2006.06.014

Ovchinnikov, V., and Karplus, M. (2012). Analysis and elimination of a bias in targeted molecular dynamics simulations of conformational transitions: application to calmodulin. J. Phys. Chem. B 116, 8584-8603. doi: $10.1021 /$ jp212634z

Page, M. G. (2012). The role of the outer membrane of Gram-negative bacteria in antibiotic resistance: Ajax' shield or Achilles' heel? Handb. Exp. Pharmacol. 211, 67-86. doi: 10.1007/978-3-642-28951-4_5

Pages, J. M., Lavigne, J. P., Leflon-Guibout, V., Marcon, E., Bert, F., Noussair, L., et al. (2009). Efflux pump, the masked side of beta-lactam resistance in Klebsiella pneumoniae clinical isolates. PLOS ONE 4:e4817. doi: 10.1371/journal.pone.0004817

Pages, J. M., Masi, M., and Barbe, J. (2005). Inhibitors of efflux pumps in Gram-negative bacteria. Trends Mol. Med. 11, 382-389. doi: 10.1016/j.molmed. 2005.06.006

Pai, H., Kim, J., Kim, J., Lee, J. H., Choe, K. W., and Gotoh, N. (2001). Carbapenem resistance mechanisms in Pseudomonas aeruginosa clinical isolates. Antimicrob. Agents Chemother. 45, 480-484. doi: 10.1128/AAC.45.2.480-484.2001

Paixao, L., Rodrigues, L., Couto, I., Martins, M., Fernandes, P., De Carvalho, C. C., et al. (2009). Fluorometric determination of ethidium bromide efflux kinetics in Escherichia coli. J. Biol. Eng. 3, 18. doi: 10.1186/1754-1611-3-18

Parr, T. R. Jr., Moore, R. A., Moore, L. V., and Hancock, R. E. (1987). Role of porins in intrinsic antibiotic resistance of Pseudomonas cepacia. Antimicrob. Agents Chemother. 31, 121-123. doi: 10.1128/AAC.31.1.121

Phan, G., Benabdelhak, H., Lascombe, M.-B., Benas, P., Rety, S., Picard, M., et al. (2010). Structural and dynamical Insights into the opening mechanism of $P$. aeruginosa OprM Channel. Structure 18, 507-517. doi: 10.1016/j.str.2010.01.018

Phillips, J. L., and Gnanakaran, S. (2015). A Data-driven approach to modeling the tripartite structure of multidrug resistance efflux pumps. Proteins 83, 43-65. doi: $10.1002 /$ prot. 24632

Piddock, L. J. (2006). Clinically relevant chromosomally encoded multidrug resistance efflux pumps in bacteria. Clin. Microbiol. Rev. 19, 382-402. doi: 10.1128/CMR.19.2.382-402.2006

Piddock, L. J., Garvey, M. I., Rahman, M. M., and Gibbons, S. (2010). Natural and synthetic compounds such as trimethoprim behave as inhibitors of efflux in Gram-negative bacteria. J. Antimicrob. Chemother. 65, 1215-1223. doi: 10.1093/jac/dkq079

Pietras, Z., Bavro, V. N., Furnham, N., Pellegrini-Calace, M., Milner-White, E. J., and Luisi, B. F. (2008). Structure and mechanism of drug efflux machinery in Gram negative bacteria. Curr. Drug Targets 9, 719-728. doi: $10.2174 / 138945008785747743$

Podnecky, N. L., Wuthiekanun, V., Peacock, S. J., and Schweizer, H. P. (2013). The BpeEF-OprC efflux pump is responsible for widespread trimethoprim resistance in clinical and environmental Burkholderia pseudomallei isolates. Antimicrob. Agents Chemother. 57, 4381-4386. doi: 10.1128/AAC. 00660-13
Poole, K. (2000). Efflux-mediated resistance to fluoroquinolones in gramnegative bacteria. Antimicrob. Agents Chemother. 44, 2233-2241. doi: 10.1128/AAC.44.9.2233-2241.2000

Poole, K. (2004). Efflux-mediated multiresistance in Gram-negative bacteria. Clin. Microbiol. Infect. 10, 12-26. doi: 10.1111/j.1469-0691.2004.00763.x

Poole, K. (2005). Efflux-mediated antimicrobial resistance. J. Antimicrob. Chemother. 56, 20-51. doi: 10.1093/jac/dki171

Poole, K. (2011). Pseudomonas aeruginosa: resistance to the max. Front. Microbiol. 2:65. doi: $10.3389 /$ fmicb.2011.00065

Poole, K. (2013). "Pseudomonas aeruginosa efflux pumps," in: Microbial Efflux Pumps, eds E. W. Yu, Q. Zhang, and M. H. Brown (Norfolk: Caister Academic Press).

Poole, K. (2014). Stress responses as determinants of antimicrobial resistance in Pseudomonas aeruginosa: multidrug efflux and more. Can. J. Microbiol. 60, 783-791. doi: 10.1139/cjm-2014-0666

Potron, A., Poirel, L., and Nordmann, P. (2015). Emerging broad-spectrum resistance in Pseudomonas aeruginosa and Acinetobacter baumannii: mechanisms and epidemiology. Int. J. Antimicrob. Agents 45, 568-585. doi: 10.1016/j.ijantimicag.2015.03.001

Pournaras, S., Maniati, M., Spanakis, N., Ikonomidis, A., Tassios, P. T., Tsakris, A., et al. (2005). Spread of efflux pump-overexpressing, non-metallobeta-lactamase-producing, meropenem-resistant but ceftazidime-susceptible Pseudomonas aeruginosa in a region with blaVIM endemicity. J. Antimicrob. Chemother. 56, 761-764. doi: 10.1093/jac/dki296

Pumbwe, L., and Piddock, L. J. (2000). Two efflux systems expressed simultaneously in multidrug-resistant Pseudomonas aeruginosa. Antimicrob. Agents Chemother. 44, 2861-2864. doi: 10.1128/AAC.44.10.2861-2864.2000

Rampersad, S. N. (2012). Multiple applications of Alamar Blue as an indicator of metabolic function and cellular health in cell viability bioassays. Sensors (Basel) 12, 12347-12360. doi: 10.3390/s120912347

Raunest, M., and Kandt, C. (2012). Locked on one side only: ground state dynamics of the outer membrane efflux duct TolC. Biochemistry 51, 1719-1729. doi: $10.1021 / \mathrm{bi} 201814 \mathrm{~s}$

Renau, T. E., Leger, R., Yen, R., She, M. W., Flamme, E. M., Sangalang, J., et al. (2002). Peptidomimetics of efflux pump inhibitors potentiate the activity of levofloxacin in Pseudomonas aeruginosa. Bioorg. Med. Chem. Lett. 12, 763-766. doi: 10.1016/S0960-894X(02)00006-9

Renau, T. E., and Lemoine, R. C. (2001). Efflux pump ihibitors to address bacterial and fungal resistance. Drugs Fut. 26, 1171-1178. doi: 10.1358/dof.2001.026.12.644122

Richmond, G. E., Chua, K. L., and Piddock, L. J. (2013). Efflux in Acinetobacter baumannii can be determined by measuring accumulation of H33342 (bisbenzamide). J. Antimicrob. Chemother. 68, 1594-1600. doi: 10.1093/jac/dkt052

Riera, E., Cabot, G., Mulet, X., Garcia-Castillo, M., Del Campo, R., Juan, C., et al. (2011). Pseudomonas aeruginosa carbapenem resistance mechanisms in Spain: impact on the activity of imipenem, meropenem and doripenem. J. Antimicrob. Chemother. 66, 2022-2027. doi: 10.1093/jac/dkr232

Riou, M., Carbonnelle, S., Avrain, L., Mesaros, N., Pirnay, J. P., Bilocq, F., et al. (2010). In vivo development of antimicrobial resistance in Pseudomonas aeruginosa strains isolated from the lower respiratory tract of Intensive Care Unit patients with nosocomial pneumonia and receiving antipseudomonal therapy. Int. J. Antimicrob. Agents. 36, 513-522. doi: 10.1016/j.ijantimicag.2010.08.005

Rosen, B. P., and Kashket, E. R. (1978). "Energetics of active transport," in: Bacterial Transport, ed. B. P. Rosen (New York: Marcel Dekker Inc.).

Ruggerone, P., Murakami, S., Pos, K. M., and Vargiu, A. V. (2013a). Rnd efflux pumps: structural information translated into function and inhibition mechanisms. Curr. Top. Med. Chem. 13, 3079-3100. doi: 10.2174/156802661 13136660220

Ruggerone, P., Vargiu, A. V., Collu, F., Fischer, N., and Kandt, C. (2013b). Molecular dynamics computer simulations of multidrug RND efflux pumps. Computat. Struct. Biotechnol. J. 5:e201302008. doi: 10.5936/csbj.201302008

Russo-Marie, F., Roederer, M., Sager, B., Herzenberg, L. A., and Kaiser, D. (1993). Beta-galactosidase activity in single differentiating bacterial cells. Proc. Natl. Acad. Sci. U.S.A. 90, 8194-8198. doi: 10.1073/pnas.90.17.8194

Ruzin, A., Keeney, D., and Bradford, P. A. (2005). AcrAB efflux pump plays a role in decreased susceptibility to tigecycline in Morganella morganii. Antimicrob. Agents Chemother. 49, 791-793. doi: 10.1128/AAC.49.2.791-793.2005 
Schlitter, J., Engels, M., Kruger, P., Jacoby, E., and Wollmer, A. (1993). Targeted molecular-dynamics simulation of conformational change - application to the T[-]R transition in insulin. Mol. Simulat. 10, 291-308. doi: $10.1080 / 08927029308022170$

Schulz, G. E. (1993). Bacterial porins: structure and function. Curr. Opin. Cell Biol. 5, 701-707. doi: 10.1016/0955-0674(93)90143-E

Schulz, R., and Kleinekathöfer, U. (2009). Transitions between closed and open conformations of TolC: the effects of ions in simulations. Biophys. J. 96, 3116-3125. doi: 10.1016/j.bpj.2009.01.021

Schulz, R., Vargiu, A. V., Collu, F., Kleinekathoefer, U., and Ruggerone, P. (2010). Functional rotation of the transporter AcrB: insights into drug extrusion from simulations. PLoS Comput. Biol. 6:e1000806. doi: 10.1371/journal.pcbi.10 00806

Schulz, R., Vargiu, A. V., Ruggerone, P., and Kleinekathöfer, U. (2011). Role of water during the extrusion of substrates by the efflux transporter AcrB. J. Phys. Chem. B 115, 8278-8287. doi: 10.1021/jp200996x

Schulz, R., Vargiu, A. V., Ruggerone, P., and Kleinekathöfer, U. (2015). Computational study of correlated domain motions in the acrb efflux transporter. BioMed. Res. Int. 12:487298 doi: 10.1155/2015/487298

Schumacher, A., Trittler, R., Bohnert, J. A., Kummerer, K., Pages, J. M., and Kern, W. V. (2007). Intracellular accumulation of linezolid in Escherichia coli, citrobacter freundii and enterobacter aerogenes: role of enhanced efflux pump activity and inactivation. J. Antimicrob. Chemother. 59, 1261-1264. doi: $10.1093 / \mathrm{jac} / \mathrm{dkl} 380$

Schweizer, H. P. (2003). Efflux as a mechanism of resistance to antimicrobials in Pseudomonas aeruginosa and related bacteria: unanswered questions. Genet. Mol. Res. 2, 48-62.

Sedgwick, E. G., and Bragg, P. D. (1996). The role of efflux systems and the cell envelope in fluorescence changes of the lipophilic cation 2-(4dimethylaminostyryl)-1-ethylpyridinium in Escherichia coli. Biochim. Biophys. Acta 1278, 205-212. doi: 10.1016/0005-2736(95)00228-6

Seeger, M. A., Schiefner, A., Eicher, T., Verrey, F., Diederichs, K., and Pos, K. M. (2006). Structural asymmetry of AcrB trimer suggests a peristaltic pump mechanism. Science 313, 1295-1298. doi: 10.1126/science.1131542

Sekiya, H., Mima, T., Morita, Y., Kuroda, T., Mizushima, T., and Tsuchiya, T. (2003). Functional cloning and characterization of a multidrug efflux pump, mexHI-opmD, from a Pseudomonas aeruginosa mutant. Antimicrob. Agents Chemother. 47, 2990-2992. doi: 10.1128/AAC.47.9.2990-2992.2003

Senn, H. M., and Thiel, W. (2009). QM/MM methods for biomolecular systems. Angew. Chem. Int. Ed. Engl. 48, 1198-1229. doi: 10.1002/anie.200802019

Sennhauser, G., Amstutz, P., Briand, C., Storchenegger, O., and Grutter, M. G. (2007). Drug export pathway of multidrug exporter AcrB revealed by Darpin inhibitors. PLoS Biol. 5:e7. doi: 10.1371/journal.pbio.0050007

Sennhauser, G., Bukowska, M. A., Briand, C., and Grutter, M. G. (2009). Crystal structure of the multidrug exporter MexB from Pseudomonas aeruginosa. J. Mol. Biol. 389, 134-145. doi: 10.1016/j.jmb.2009.04.001

Sgrignani, J., and Magistrato, A. (2013). First-principles modeling of biological systems and structure-based drug-design. Curr. Comput. Aided Drug Des. 9, $15-34$.

Shapiro, H. M. (2000). Membrane potential estimation by flow cytometry. Methods 21, 271-279. doi: 10.1006/meth.2000.1007

Steinbrecher, T., and Elstner, M. (2013). QM and QM/MM simulations of proteins. Methods Mol. Biol. 924, 91-124. doi: 10.1007/978-1-62703-017-5_5

Steinbrecher, T., Prock, S., Reichert, J., Wadhwani, P., Zimpfer, B., Bürck, J., et al. (2012). Peptide-lipid interactions of the stress-response peptide TisB that induces bacterial persistence. Biophys. J. 103, 1460-1469. doi: 10.1016/j.bpj.2012.07.060

Sterpone, F., Ceccarelli, M., and Marchi, M. (2001). Dynamics of hydration in hen egg white lysozyme. J. Mol. Biol. 311, 409-419. doi: 10.1006/jmbi.2001.4860

$\mathrm{Su}, \mathrm{C}$. C., and Yu, E. W. (2007). Ligand-transporter interaction in the AcrB multidrug efflux pump determined by fluorescence polarization assay. FEBS Lett. 581, 4972-4976. doi: 10.1016/j.febslet.2007.09.035

Sugawara, E., Nagano, K., and Nikaido, H. (2010). Factors affecting the folding of Pseudomonas aeruginosa OprF porin into the one-domain open conformer. MBio 1:e00228-10. doi: 10.1128/mBio.00228-10

Sugawara, E., Nestorovich, E. M., Bezrukov, S. M., and Nikaido, H. (2006). Pseudomonas aeruginosa porin OprF exists in two different conformations. J. Biol. Chem. 281, 16220-16229. doi: 10.1074/jbc.M600680200
Sun, J., Deng, Z., and Yan, A. (2014). Bacterial multidrug efflux pumps: mechanisms, physiology and pharmacological exploitations. Biochem. Biophys. Res. Commun. 453, 254-267. doi: 10.1016/j.bbrc.2014.05.090

Symmons, M., Bokma, E., Koronakis, E., Hughes, C., and Koronakis, V. (2009). The assembled structure of a complete tripartite bacterial multidrug efflux pump. Proc. Natl. Acad. Sci. U.S.A. 106, 7173-7178. doi: 10.1073/pnas.09006 93106

Takatsuka, Y., Chen, C., and Nikaido, H. (2010). Mechanism of recognition of compounds of diverse structures by the multidrug efflux pump AcrB of Escherichia coli. Proc. Natl. Acad. Sci. U.S.A. 107, 6559-6565. doi: 10.1073/pnas.1001460107

Thanassi, D. G., Cheng, L. W., and Nikaido, H. (1997). Active efflux of bile salts by Escherichia coli. J. Bacteriol. 179, 2512-2518.

Tikhonova, E. B., Wang, Q., and Zgurskaya, H. I. (2002). Chimeric analysis of the multicomponent multidrug efflux transporters from gram-negative bacteria. J. Bacteriol. 184, 6499-6507. doi: 10.1128/JB.184.23.6499-65 07.2002

Tikhonova, E. B., Yamada, Y., and Zgurskaya, H. I. (2011). Sequential mechanism of assembly of multidrug efflux pump AcrAB-TolC. Chem. Biol. 18, 454-463. doi: 10.1016/j.chembiol.2011.02.011

Trepout, S., Taveau, J. C., Benabdelhak, H., Granier, T., Ducruix, A., Frangakis, A. S., et al. (2010). Structure of reconstituted bacterial membrane efflux pump by cryo-electron tomography. Biochim. Biophys. Acta 1798, 1953-1960. doi: 10.1016/j.bbamem.2010.06.019

Vaara, M. (1992). Agents that increase the permeability of the outer membrane. Microbiol. Rev. 56, 395-411.

Vaccaro, L., Koronakis, V., and Sansom, M. S. P. (2006). Flexibility in a drug transport accessory protein: molecular dynamics simulations of MexA. Biophys. J. 91, 558-564. doi: 10.1529/biophysj.105.080010

Vaccaro, L., Scott, K. A., and Sansom, M. S. P. (2008). Gating at both ends and breathing in the middle: conformational dynamics of TolC. Biophys. J. 95, 5681-5691. doi: 10.1529/biophysj.108.136028

Van Bambeke, F., Pagès, J.-M., and Lee, V. J. (2010). Inhibitors of bacterial efflux pumps as adjuvant in antibacterial therapy and diagnostic tools for detection of resistance by efflux. Front. Anti Infect. Drug Discov. 1: 138-175.

van den Berg van Saparoea, H. B., Lubelski, J., Van Merkerk, R., Mazurkiewicz, P. S., and Driessen, A. J. (2005). Proton motive force-dependent Hoechst 33342 transport by the $\mathrm{ABC}$ transporter LmrA of Lactococcus lactis. Biochemistry 44, 16931-16938. doi: 10.1021/bi051497y

Vargiu, A. V., Collu, F., Schulz, R., Pos, K. M., Zacharias, M., Kleinekathöfer, U., et al. (2011). Effect of the F610A mutation on substrate extrusion in the AcrB transporter: explanation and rationale by molecular dynamics simulations. J. Am. Chem. Soc. 133, 10704-10707. doi: 10.1021/ja20 2666x

Vargiu, A. V., and Nikaido, H. (2012). Multidrug binding properties of the AcrB efflux pump characterized by molecular dynamics simulations. Proc. Natl. Acad. Sci. U.S.A. 109, 20637-20642. doi: 10.1073/pnas.1218348109

Vargiu, A. V., Ruggerone, P., Opperman, T. J., Nguyen, S. T., and Nikaido, H. (2014). Molecular mechanism of Mbx2319 inhibition of Escherichia coli AcrB multidrug efflux pump and comparison with other inhibitors. Antimicrob. Agents Chemother. 58, 6224-6234. doi: 10.1128/AAC. 03283-14

Verchère, A., Dezi, M., Adrien, V., Broutin, I., and Picard, M. (2015). In vitro transport activity of the fully assembled MexAB-OprM efflux pump from Pseudomonas aeruginosa. Nat. Commun. 6, 6890. doi: 10.1038/ ncomms7890

Verchère, A., Dezi, M., Broutin, I., and Picard, M. (2014). In vitro investigation of the MexAB efflux pump from Pseudomonas aeruginosa. J. Vis. Exp. 84:e50894. doi: $10.3791 / 50894$

Vidal-Aroca, F., Meng, A., Minz, T., Page, M. G., and Dreier, J. (2009). Use of resazurin to detect mefloquine as an efflux-pump inhibitor in Pseudomonas aeruginosa and Escherichia coli. J. Microbiol. Methods 79, 232-237. doi: 10.1016/j.mimet.2009.09.021

Visalli, M. A., Murphy, E., Projan, S. J., and Bradford, P. A. (2003). AcrAB multidrug efflux pump is associated with reduced levels of susceptibility to tigecycline (GAR-936) in Proteus mirabilis. Antimicrob. Agents Chemother. 47, 665-669. doi: 10.1128/AAC.47.2.665-669.2003 
Viveiros, M., Martins, A., Paixao, L., Rodrigues, L., Martins, M., Couto, I., et al. (2008). Demonstration of intrinsic efflux activity of Escherichia coli K-12 AG100 by an automated ethidium bromide method. Int. J. Antimicrob. Agents 31, 458-462. doi: 10.1016/j.ijantimicag.2007.12.015

Walmsley, A. R., Martin, G. E., and Henderson, P. J. (1994). 8-Anilino-1naphthalenesulfonate is a fluorescent probe of conformational changes in the D-galactose- $\mathrm{H}^{+}$symport protein of Escherichia coli. J. Biol. Chem. 269, 17009-17019.

Walsh, F., and Amyes, S. G. (2007). Carbapenem resistance in clinical isolates of Pseudomonas aeruginosa. J. Chemother. 19, 376-381. doi: 10.1179/joc.2007.19.4.376

Wang, B., Weng, J., Fan, K., and Wang, W. (2012). Interdomain flexibility and $\mathrm{pH}$-Induced conformational changes of AcrA revealed by molecular dynamics simulations. J. Phys. Chem. B 116, 3411-3420. doi: 10.1021/jp212221v

Watkins, W. J., Landaverry, Y., Leger, R., Litman, R., Renau, T. E., Williams, N., et al. (2003). The relationship between physicochemical properties, in vitro activity and pharmacokinetic profiles of analogues of diamine-containing efflux pump inhibitors. Bioorg. Med. Chem. Lett. 13, 4241-4244. doi: 10.1016/j.bmcl.2003.07.030

Webber, M. A., and Piddock, L. J. (2003). The importance of efflux pumps in bacterial antibiotic resistance. J. Antimicrob. Chemother. 51, 9-11. doi: $10.1093 / \mathrm{jac} / \mathrm{dkg} 050$

Wehmeier, C., Schuster, S., Fahnrich, E., Kern, W. V., and Bohnert, J. A. (2009). Site-directed mutagenesis reveals amino acid residues in the Escherichia coli RND efflux pump AcrB that confer macrolide resistance. Antimicrob. Agents Chemother. 53, 329-330. doi: 10.1128/AAC.00921-08

Welch, A., Awah, C. U., Jing, S., Van Veen, H. W., and Venter, H. (2010). Promiscuous partnering and independent activity of MexB, the multidrug transporter protein from Pseudomonas aeruginosa. Biochem. J. 430, 355-364. doi: 10.1042/BJ20091860

Xu, X. H. N., Brownlow, W., Huang, S., and Chen, J. (2003). Single-molecule detection of efflux pump machinery in Pseudomonas aeruginosa. Biochem. Biophys. Res. Commun. 305, 79-86. doi: 10.1016/S0006-291X(03)00692-2

Xu, Y., Lee, M., Moeller, A., Song, S., Yoon, B.-Y., Kim, H.-M., et al. (2011). Funnel-like hexameric assembly of the periplasmic adapter protein in the tripartite multidrug efflux pump in gram-negative bacteria. J. Biol. Chem. 286, 17910-17920. doi: 10.1074/jbc.M111.238535

Yamaguchi, A., Nakashima, R., and Sakurai, K. (2015). Structural basis of RND-type multidrug exporters. Front. Microbiol. 6:327. doi: 10.3389/fmicb.2015.00327

Yamaguchi, A., Udagawa, T., and Sawai, T. (1990). Transport of divalent cations with tetracycline as mediated by the transposon Tn10-encoded tetracycline resistance protein. J. Biol. Chem. 265, 4809-4813.
Yang, N. C., and Hu, M. L. (2004). A fluorimetric method using fluorescein di-beta-D-galactopyranoside for quantifying the senescenceassociated beta-galactosidase activity in human foreskin fibroblast Hs68 cells. Anal. Biochem. 325, 337-343. doi: 10.1016/j.ab.2003. 11.012

Yao, X.-Q., Kimura, N., Murakami, S., and Takada, S. (2013). Drug uptake pathways of multidrug transporter AcrB studied by molecular simulations and site-directed mutagenesis experiments. J. Am. Chem. Soc. 135, 7474-7485. doi: $10.1021 / \mathrm{ja} 310548 \mathrm{~h}$

Yoshida, K., Nakayama, K., Ohtsuka, M., Kuru, N., Yokomizo, Y., Sakamoto, A., et al. (2007). MexAB-OprM specific efflux pump inhibitors in Pseudomonas aeruginosa. Part 7: highly soluble and in vivo active quaternary ammonium analogue D13-9001, a potential preclinical candidate. Bioorg. Med. Chem. 15, 7087-7097. doi: 10.1016/j.bmc.2007.07.039

Yoshimura, F., and Nikaido, H. (1982). Permeability of Pseudomonas aeruginosa outer membrane to hydrophilic solutes. J. Bacteriol. 152, 636-642.

Zechini, B., and Versace, I. (2009). Inhibitors of multidrug resistant efflux systems in bacteria. Recent Patents Anti Infect. Drug Discov. 4, 37-50. doi: $10.2174 / 157489109787236256$

Zgurskaya, H. I., and Nikaido, H. (1999). Bypassing the periplasm: reconstitution of the AcrAB multidrug efflux pump of Escherichia coli. Proc. Natl. Acad. Sci. U.S.A. 96, 7190-7195. doi: 10.1073/pnas.96. 13.7190

Zhanel, G. G., Decorby, M., Adam, H., Mulvey, M. R., Mccracken, M., LagaceWiens, P., et al. (2010). Prevalence of antimicrobial-resistant pathogens in Canadian hospitals: results of the Canadian Ward Surveillance Study (Canward 2008). Antimicrob. Agents Chemother. 54, 4684-4693. doi: 10.1128/AAC. 00469-10

Zhou, T., Huang, D., and Caflisch, A. (2010). Quantum mechanical methods for drug design. Curr. Top. Med. Chem. 10, 33-45. doi: 10.2174/1568026 10790232242

Conflict of Interest Statement: Jürg Dreier is employee of Basilea Pharmaceutica International Ltd. Paolo Ruggerone declares that the research was conducted in the absence of any commercial or financial relationships that could be construed as a potential conflict of interest.

Copyright (c) 2015 Dreier and Ruggerone. This is an open-access article distributed under the terms of the Creative Commons Attribution License (CC BY). The use, distribution or reproduction in other forums is permitted, provided the original author(s) or licensor are credited and that the original publication in this journal is cited, in accordance with accepted academic practice. No use, distribution or reproduction is permitted which does not comply with these terms. 